\title{
Structural Biology and Regulation of Protein Import into the Nucleus
}

\author{
Mary Christie ${ }^{1,2, \dagger}$, Chiung-Wen Chang ${ }^{3,4, \dagger}$, Gergely Róna ${ }^{5,6, \dagger}$, Kate M. Smith ${ }^{7, \dagger}$, \\ Alastair G. Stewart ${ }^{8}$, Agnes A.S. Takeda ${ }^{9}$, Marcos R.M. Fontes ${ }^{9}$, Murray Stewart ${ }^{3,10}$, \\ Beáta G. Vértessy ${ }^{5,6}$, Jade K. Forwood ${ }^{7}$ and Bostjan Kobe ${ }^{3}$ \\ 1 - The Garvan Institute of Medical Research, 384 Victoria Street, Darlinghurst, NSW 2010, Australia \\ 2 - St Vincent's Clinical School, University of New South Wales Faculty of Medicine, Darlinghurst, NSW 2010, Australia \\ 3 - School of Chemistry and Molecular Biosciences, Institute for Molecular Bioscience and Australian Infectious Diseases Research \\ Centre, University of Queensland, Brisbane, QLD 4072, Australia \\ 4 - Verna and Marrs McLean Department of Biochemistry and Molecular Biology, Baylor College of Medicine, Houston, \\ TX 77030, USA \\ 5 - Institute of Enzymology, Research Centre for Natural Sciences, Hungarian Academy of Sciences, Budapest H-1117, Hungary \\ 6 - Department of Applied Biotechnology and Food Sciences, Budapest University of Technology and Economics, \\ Budapest $\mathrm{H}-1111$, Hungary \\ 7 - School of Biomedical Sciences, Charles Sturt University, Wagga Wagga, NSW 2650, Australia \\ 8 - School of Molecular Bioscience, The University of Sydney, Sydney, NSW 2006, Australia \\ 9 - Department of Physics and Biophysics, Institute of Biosciences, Universidade Estadual Paulista, Botucatu, \\ São Paulo 18618-000, Brazil \\ 10 - MRC Laboratory of Molecular Biology, Francis Crick Avenue, Cambridge Biomedical Campus, Cambridge CB2 OQH, \\ United Kingdom
}

Correspondence to Bostjan Kobe: b.kobe@uq.edu.au

http://dx.doi.org/10.1016/j.jmb.2015.10.023

Edited by D. Görlich

\begin{abstract}
Proteins are translated in the cytoplasm, but many need to access the nucleus to perform their functions. Understanding how these nuclear proteins are transported through the nuclear envelope and how the import processes are regulated is therefore an important aspect of understanding cell function. Structural biology has played a key role in understanding the molecular events during the transport processes and their regulation, including the recognition of nuclear targeting signals by the corresponding receptors. Here, we review the structural basis of the principal nuclear import pathways and the molecular basis of their regulation. The pathways involve transport factors that are members of the $\beta$-karyopherin family, which can bind cargo directly (e.g., importin- $\beta$, transportin-1, transportin-3, importin-13) or through adaptor proteins (e.g., importin- $\alpha$, snurportin-1, symportin-1), as well as unrelated transport factors such as Hikeshi, involved in the transport of heat-shock proteins, and NTF2, involved in the transport of RanGDP. Solenoid proteins feature prominently in these pathways. Nuclear transport factors recognize nuclear targeting signals on the cargo proteins, including the classical nuclear localization signals, recognized by the adaptor importin- $\alpha$, and the PY nuclear localization signals, recognized by transportin-1. Post-translational modifications, particularly phosphorylation, constitute key regulatory mechanisms operating in these pathways.
\end{abstract}

(C) 2015 Elsevier Ltd. All rights reserved.

\section{Overview of Nuclear Import Pathways}

The nucleus is separated from the cytoplasm by a double membrane and houses the genetic material and the transcriptional apparatus, separating it from the translational and metabolic machinery in the cytoplasm of eukaryotic cells. The key to this separation is the corresponding ability to regulate transport across the nuclear envelope. This transport occurs through nuclear pore complexes (NPCs), 
huge macromolecular structures that span both nuclear membranes and that are built from multiple copies of a large number of proteins termed nucleoporins (Nups) [1,2] (see reviews by Beck, Schwartz, Lemke and Gorlich in this issue). The NPC has a large enough channel to allow proteins smaller than $\sim 40 \mathrm{kDa}$ to passively diffuse through it; however, most, if not all, proteins with functions in the nucleus use active carrier-mediated transport. Translocation of proteins through NPCs requires additional carrier proteins or transport factors. Many of these carriers belong to the $\beta$-karyopherin ( $\beta$-Kap) (or importin- $\beta$, $\operatorname{Imp} \beta$ ) superfamily. All $\beta$-Kap family members are built from tandem HEAT repeats (named after huntingtin, elongation factor 3 , protein phosphatase $2 \mathrm{~A}$ and TOR1 [3]), each of which contains 40-45 amino acids that form two antiparallel $\alpha$-helices (designated $A$ and $B$ ) linked by a loop. This repetitive structure places $\beta$-Kaps in the solenoid protein category [4], which features prominently among proteins involved in nucleocytoplasmic transport (Fig. 1).

An additional key component of nuclear transport pathways is the small GTPase Ran. Ran cycles between GDP- and GTP-bound states [5], and the state of the bound nucleotide is determined by Ran regulatory proteins, including RanGEF (Ran guanine nucleotide exchange factor, also called RCC1, regulator of chromosome condensation 1, or Prp20p in yeast) [6] and RanGAP (Ran GTPase-activating protein, Rna1p in yeast) [7]. Like other members of the Ras-family GTPases, Ran is composed of a small G-domain and contains two surface loops, termed switch-I and switch-II, which change conformation depending on the nucleotide-bound state of the protein. The RanGDP/GTP gradient, generated by RanGEF and RanGAP being located in the nucleus and the cytoplasm, respectively, establishes directionality in nucleocytoplasmic transport pathways. As a result, import receptors that bind cargo in the cytoplasm can release it in the nucleus through binding RanGTP [5,8-10], whereas export receptors can bind cargo in the nucleus through simultaneous binding of RanGTP and can release it in the cytoplasm after GTP hydrolysis is triggered.

Whether a protein localizes to the nucleus is usually determined by nuclear targeting signals. The first nuclear targeting signal discovered, and the best characterized, is the classical nuclear localization sequence (cNLS) recognized by the protein importin- $\alpha$ (Impa) (karyopherin- $\alpha$ ). Impa is an adaptor protein that links the cargo to a carrier protein that actually takes the cargo through the NPC; its specific carrier protein is importin- $\beta$ (Imp $\beta 1$ ) (karyopherin- $\beta 1)$ [11]. Impa is also a solenoid but is built from armadillo (ARM) repeats [12,13]. Some proteins also have nuclear export signals (NESs) $[14,15]$ and can shuttle in and out of the nucleus.

The members of the $\beta$-Kap family transport cargoes by binding the nuclear localization signal (NLS) either

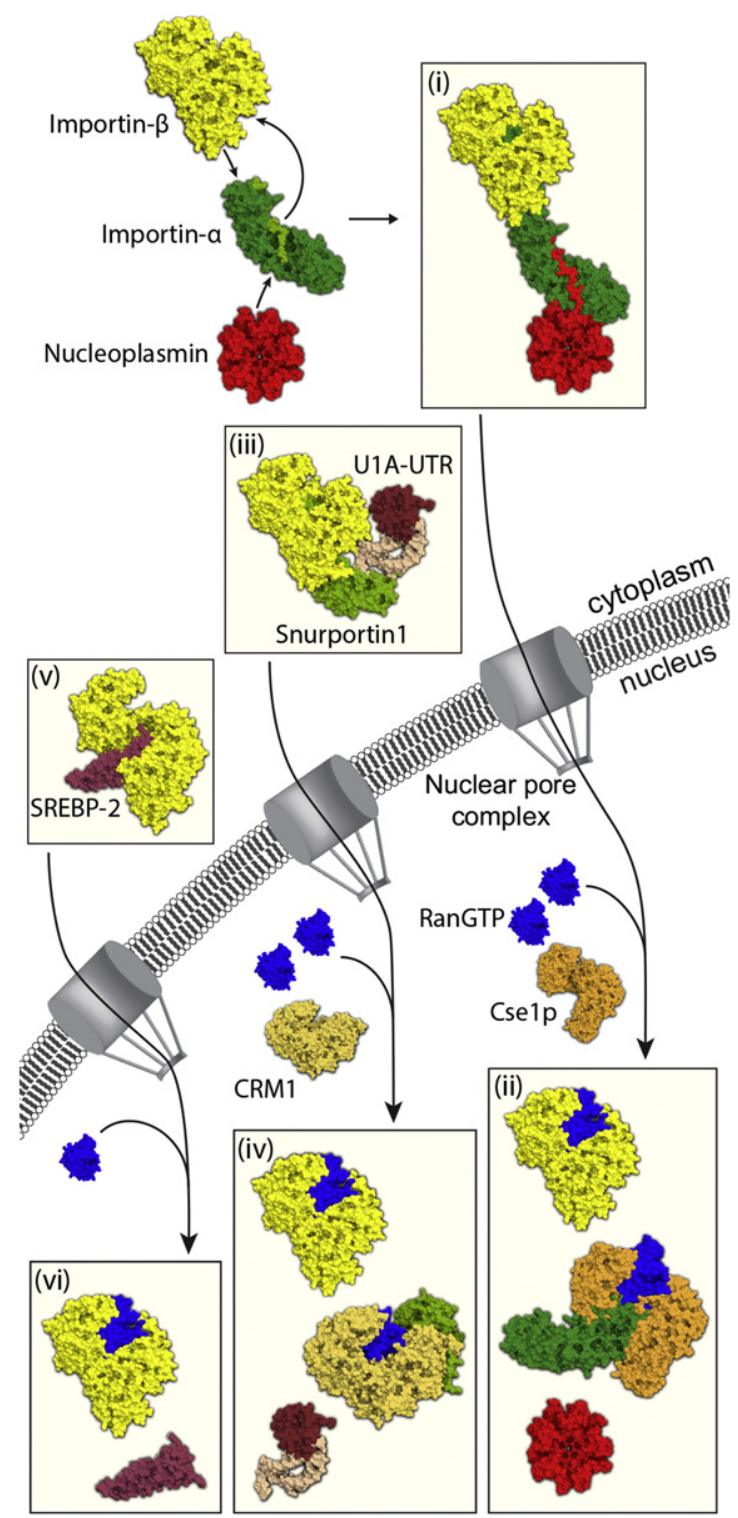

Fig. 1. Overview of the main nuclear import pathways. Schematic illustration of three different nuclear import pathways. (i and ii) Classic import pathway; cargo (nucleoplasmin, shown in red; PDB entry $1 \mathrm{~K} 5 \mathrm{~J}$ ), Impa (shown in green; PDB entries $1 \mathrm{IAL}$ and 1EE5) and Imp $\beta 1$ (shown in yellow; PDB entry 1QGK) form a ternary complex before translocating across the membrane via the nuclear pore (shown in gray); RanGTP (shown in blue; $\mathrm{PDB}$ entry 2BKU) and Cse1p [or CAS] (shown in orange; PDB entry 1WA5) dissociate the complex and release the cargo. (iii and iv) Snurportin-1 import pathway; cargo (U1A-UTR, shown in red and wheat; PDB entry 1AUD), snurportin-1 (shown in green; PDB entry 1UKL) and Imp $\beta 1$ translocate across the membrane; RanGTP and CRM1 (shown in orange; PDB entry 3GJX) release the cargo. ( $v$ and vi) Import pathway involving direct cargo binding to Imp $\beta 1$; cargo (SREBP2, shown in red; PDB entry $1 \mathrm{UKL}$ ) and Imp $\beta 1$ form a binary complex before translocating across the membrane; RanGTP dissociates the complex. 
directly or through adaptor molecules such as Impa or snurportin-1 (Fig. 1). There are $20 \beta$-Kap family members in humans, 10 of which mediate transport of macromolecules into the nucleus, 7 translocate macromolecules from the nucleus to the cytoplasm, 2 have been shown to mediate translocation in both directions and 1 member remains to be characterized. The yeast genome codes for $14 \beta$-Kaps. The reason for the large repertoire of nuclear import receptors within cells remains to be fully elucidated; in part, it can be attributed to the requirement of cells to translocate hundreds of quite disparate macromolecules across the nuclear envelope. It is emerging that different $\beta$-Kap family members recognize different classes of cargoes, and moreover, tissue-specific expression of family members may differentially localize cargoes within different cell types. However, it also appears that there is some redundancy in this system, with several $\beta$-Kap receptors able to recognize the same cargoes [16].

Structural biology has played an important role in deciphering the molecular events required for nuclear import. Here, we review the available structural information on different nuclear import pathways and the associated determinants of specificity in these pathways, and we also review the regulatory mechanisms acting on the import processes. The main pathways are summarized in Fig. 1, and the representative protein structures are listed in Table 1. The structures of nuclear export complexes are reviewed by Matsuura [256].

\section{Nuclear Import Mediated by $\beta$-Kap Family Members}

\section{Overview of the $\beta$-Kap family}

The members of the $\beta$-Kap family are highly conserved across eukaryotes, reflecting their critical cellular function. A higher degree of similarity is often observed between orthologues in different species than between paralogues in the same species [17], and although there is low sequence identity among family members (Supplementary Table 1), they display a high degree of structural similarity. Many HEAT repeats in the $\beta$-Kap family display conserved

Table 1. Structurally characterized transport receptors involved in protein import into the nucleus.

\begin{tabular}{|c|c|c|c|}
\hline $\begin{array}{l}\text { Transport receptor } \\
\text { (abbreviation) }\end{array}$ & Other names & $\begin{array}{l}\text { Transport } \\
\text { direction }\end{array}$ & Representative available structures (PDB entry) \\
\hline Importin- $\beta$ (Imp $\beta 1)$ & $\begin{array}{c}\text { Importin-90, karyopherin } \beta-1 \text {, nuclear } \\
\text { factor p97, pore-targeting complex } \\
\text { 97-kDa subunit, (PTAC97), Kap95p } \\
\text { (yeast; ylmp } \beta 1 \text { ) }\end{array}$ & Import & 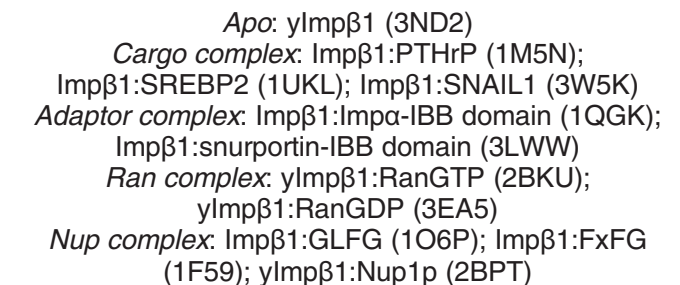 \\
\hline Transportin-1 (Trn1) & $\begin{array}{l}\text { Importin- } \beta 2 \text {, karyopherin- } \beta 2 \text {, } \\
\text { M9 region interaction protein } \\
\text { (MIP); Kap104p (yeast; yTrn1) }\end{array}$ & Import & $\begin{array}{c}\text { Apo: Trn1 (2QMR, 2Z5J) } \\
\text { Cargo complex: Trn1:hnRNPA1 M9 NLS (2H4M); } \\
\text { Trn1:Tap NLS (2Z5K); Trn1:hnRNP D NLS (2Z5N); } \\
\text { Trn1:hnRNP M NLS (2OT8); Trn1:JKTBP NLS (2Z5O); } \\
\text { Trn1:FUS NLS (4FQ3); Trn1:Nab2 NLS (4JLQ); } \\
\text { Trn1:HCC1 (4OO6) } \\
\text { Ran complex: Trn1:Ran (1QBK) }\end{array}$ \\
\hline Transportin-3 (Trn3) & $\begin{array}{l}\text { Importin-12, } \\
\text { Transportin-SR; Kap111p (yeast, yTrn3) }\end{array}$ & Import & $\begin{array}{c}\text { Apo: Trn3 (4COP) } \\
\text { Cargo complex: Trn3:ASF/SF2 (4C0O) } \\
\text { Ran complex: Trn3:Ran (4COQ) }\end{array}$ \\
\hline Importin-13 (Imp13) & $\begin{array}{c}\text { Ran-binding protein } 13 \text { (RanBP13), } \\
\text { karyopherin-13 (Kap13) }\end{array}$ & Bi-directional & $\begin{array}{c}\text { Apo: Imp13 (3ZKV) } \\
\text { Cargo complex: Imp13:Mago-Y14 (2X1G); } \\
\text { Imp13:UBC9 (2XWU) } \\
\text { Ran complex: Imp13:Ran (2X19) }\end{array}$ \\
\hline Importin- $\alpha$ (Impa) & $\begin{array}{l}\text { Karyopherin- } \alpha(\text { Kapa); Kap60p } \\
\text { (yeast; ylmpa) }\end{array}$ & Import & $\begin{array}{c}\text { Apo: Impa (1IAL); Supplementary Table } 3 \\
\text { Cargo complex: Impa:SV40-TAg NLS (1EJL, 1BK6); } \\
\text { Impa:nucleoplasmin NLS (1EE5, 3UL1); Impa:PB2 } \\
\text { (2JDQ); Impa:CBP80 (3FEY); Impa:VP24 (4U2X); } \\
\text { Supplementary Table } 2 \\
\text { Nup complex: ylmpa:Nup2p (2C1T); Impa:Nup50 } \\
\text { (2C1M); Supplementary Table 2 }\end{array}$ \\
\hline Snurportin-1 & & Import & $\begin{array}{l}\text { Cargo complex: snurportin-1 m3G-cap-binding } \\
\text { domain:m3GpppG-cap dinucleotide (1XK5) }\end{array}$ \\
\hline Symportin-1 & Syo1 (synchronized import) & Import & $\begin{array}{c}\text { Apo: Syo1 (4GMO) } \\
\text { Cargo complex: Syo1:RpL5:RpL11 (5AFF) }\end{array}$ \\
\hline Hikeshi & & Import & Apo: Hikeshi (3WVZ) \\
\hline
\end{tabular}


Asp and Arg residues at positions 19 and 25 of the repeat, respectively, which form hydrogen $(\mathrm{H})$-bonding ladders [18]. Similarities have been described between HEAT and ARM repeats, particularly for the conserved residues that form the hydrophobic cores [18-20]. The solenoid structure of these proteins (up to 20 repeats within members of the $\beta$-Kap family) enables variation in the helicoidal curvature through cumulative subtle changes throughout the protein $[4,21]$.

\section{Impß1-mediated nuclear import}

Imp $\beta 1$ (Kap95p in yeast, termed ylmp $\beta 1$ here) was the first member of the $\beta$-Kap family to be characterized structurally and has been crystallized in complex with a number of cargo molecules, Ran and Nups (Fig. 2). The first structure corresponded to Imp $\beta 1$ bound to the importin- $\beta$ binding (IBB) domain of Impa [22]. Imp $\beta 1$ was shown to contain 19 HEAT repeats arranged in a superhelix, forming a convex face (formed by the A-helices of each repeat) and a concave face (formed by the B-helices each repeat). The majority of the $\operatorname{Imp} \beta 1$ interactions with its binding partners occur on the concave (B-helix) face, and the competition for these sites by Ran, Impa and various cargo proteins forms the foundation for the mechanisms of assembly, disassembly and translocation. The structural flexibility of the $\operatorname{Imp} \beta 1$ structure is essential for its function [21].

\section{Apo-Impß1}

The crystal structure of apo-ylmp $\beta 1$ revealed that the 19 HEAT repeats are arranged in a tightly coiled and compact conformation [21] (Fig. 2). This tightly wound structure is mediated by HEAT repeats 2 and 4 (residues $S^{74}$ and $D^{167}$, respectively), interacting with HEAT repeat 17 residues $R^{696}, E^{737}, N^{738}$ and $\mathrm{G}^{739}$. The interaction interface has a total buried surface area of only $306 \AA^{2}$, which is considerably smaller than most protein:protein interactions, indicating that there is a relatively small energy requirement to distort the flexible ylmp $\beta 1$. This is supported by small-angle X-ray scattering analysis [21], which indicates that ylmp $\beta 1$ exists in multiple

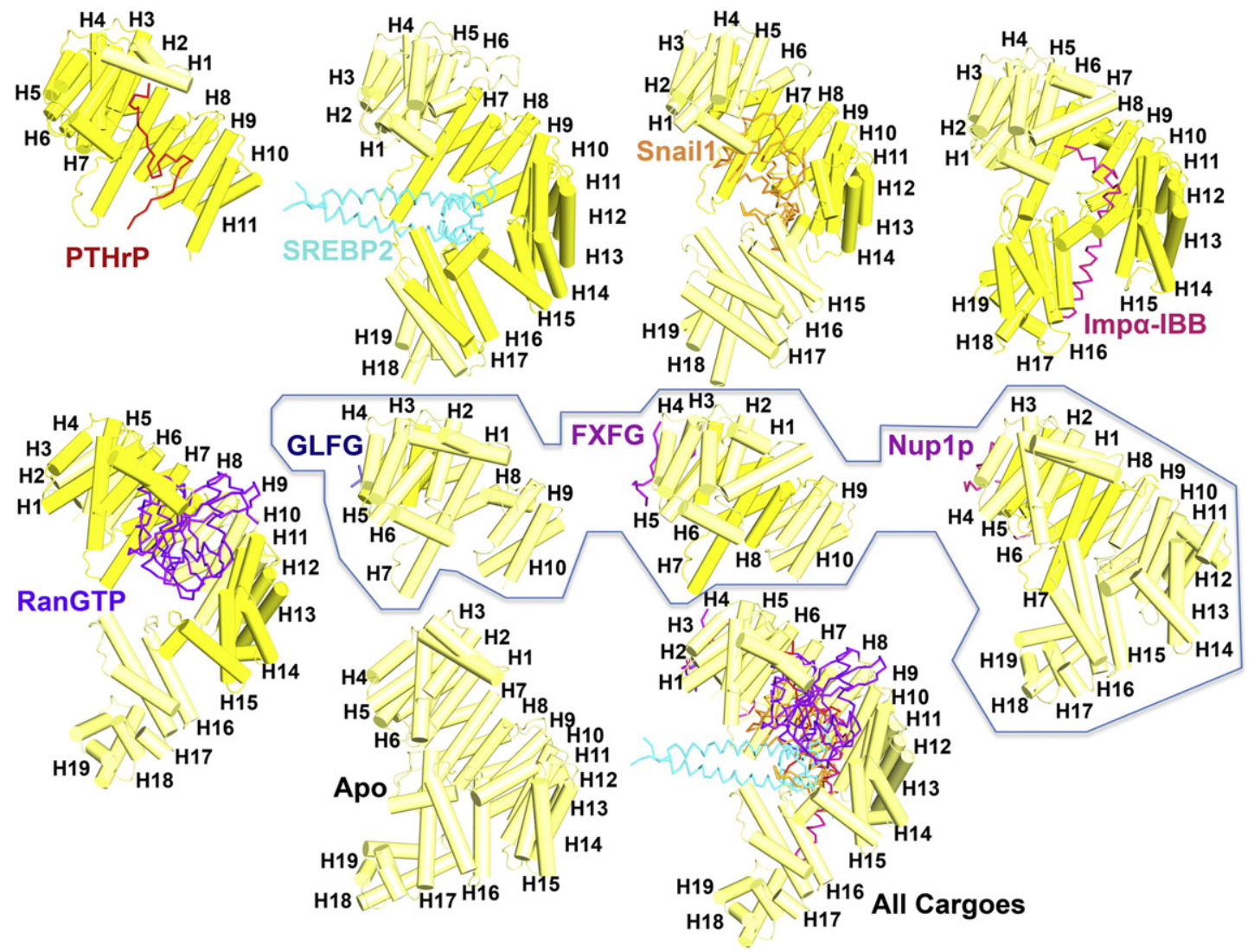

Fig. 2. Structures of Impß1. PDB entries: PTHrP complex (1M5N), SREBP2 complex (1UKL), Snail1 complex (3W5K), Impa:IBB complex (1QGK), RanGTP complex (2BKU), GLFG complex (1O6P), FxFG complex (1F59), Nup1p complex (2BPT) and apo (3ND2). The HEAT repeats involved in cargo binding are highlighted in dark yellow. A representative Imp $\beta 1$ from Imp $\beta 1$ :Ran complex has all cargoes overlaid. 
conformations in solution, and the vast array of functions it performs are achieved by taking advantage of cumulative small structural changes that efficiently allow the transition between various conformations as internal energy is stored by continuous flexing.

\section{Imp $\beta$ 1:cargo interactions}

A number of cargo proteins are recognized directly by $\operatorname{Imp} \beta 1$. The structurally characterized examples include the parathyroid hormone-related protein PTHrP [23], the sterol regulatory element-binding protein SREBP2 [24] and the zinc finger protein SNAl1 (Snail1) [25] (Fig. 2). The regions on Imp $\beta 1$ to which they bind overlap, implying that only one cargo can bind at a time.

Imp $\beta 1$ binds PTHrP using B-helices spanning HEAT repeats $2-11$ at three distinct binding surfaces. The N-terminal region of the PTHrP NLS (residues 67-79) binds to HEAT repeats $2-7$, the central region (residues $80-86$ ) binds HEAT repeats 8-10 and the C-terminal residues (residues 87-93) bind HEAT repeats 8-11. Overall, the PTHrP NLS, when bound to Imp $\beta 1$, exists in an extended conformation and buries $1027 \AA^{2}$ of surface area.

The crystal structure of the SREBP2 NLS bound to Imp $\beta 1$ revealed a helix-loop-helix structure for the cargo (SREBP2 residues 343-403) [24]. To accommodate this binding, Imp $\beta 1$ adopts a more open conformation and engages more of the C-terminal HEAT repeats, compared to the PTHrP complex. SREBP2 binds through the B-helices of HEAT repeats $7-17$. The two long helices present in repeats 7 and 17 bind SREBP2 rather similar to chopsticks. A notable difference to PTHrP is that, rather than salt bridges dominating the interactions, SREBP2 involves more hydrophobic interactions. This interaction buries $1355 \AA^{2}$ of surface area.

To bind four zinc finger domains (ZF) of Snail1 (residues 151-264), Imp $\beta 1$ uses the B-helices of HEAT repeats 5-14, including the acidic loop in HEAT repeat 8. Unlike other cargo complexes, Imp $\beta 1$ adopts a less curved structure to accommodate the bulky snail-like NLS conformation. The ZF1 domain (residues 151-176) acts as the "head", where the N-terminal $\alpha$-helix (residues 164-176) is bound within a cleft on Imp $\beta 1$ formed from HEAT repeats $9-11$. The local conformation is stabilized by a hydrophobic interaction between $\mathrm{L}^{166}$ of Snail 1 and $\mathrm{P}^{440}$ of Imp $\beta 1$. The Snail1 "shell" is composed of the three domains ZF2-ZF4, which form a compact and tight interaction with Imp $\beta 1$; the ZF2 domain (residues 177-202) forms $\mathrm{H}$-bonds with HEAT repeats 13 and 14; the $\alpha$-helix within the ZF3 domain (residues 203-230) arranges antiparallel with the inner Imp $\beta 1$ a-helix of HEAT repeat 6 ; and the ZF4 domain (residues 231-264) interacts with residues in HEAT repeats 7 and 9 . In the five C-terminal residues that represent the tail, $C^{262}$ and $R^{264}$ bind to HEAT repeat 10. Overall, Snail1 binds through 15 intermolecular interactions in the B-helices of HEAT repeats 5-14 that bury $2205 \AA^{2}$ of surface area [25].

Although the HEAT repeats used to bind the three cargo molecules (5-14 for Snail1, 2-11 for PTHrP and 7-17 for SREBP2) overlap, the binding mechanism in each is distinctly different. The interfaces overlap with the region binding RanGTP, suggesting that, regardless of the binding mechanism, the structural requirements include a large contact area and the ability for the complex to be disassembled by RanGTP binding upon entry to the nucleus.

\section{Imp $\beta$ 1:adaptor interactions}

The Imp $\beta 1$ interactions with adaptor molecules Impa and snurportin-1 have been characterized structurally (Fig. 2; reviewed in Ref. [26]). In both cases, Imp $\beta 1$ forms a closed conformation, wrapping tightly around the IBB domains of these adaptors, forming an array of salt-bridge interactions through HEAT repeats 7-19. The N-terminal residues of Impa (aIBB) (residues 11-23) and snurportin (sIBB) (residues 25-40) IBB domains mediate interactions with the HEAT repeats $7-11$, whereas the $\mathrm{C}$-terminal $\alpha$-helical regions of aIBB (residues 24-51) and sIBB (residues 41-65) bind through HEAT repeats $12-19$. Although there are common HEAT repeats involved in binding adaptor and cargo molecules, the overall mechanism of binding is distinctly different between these two groups. Not only is the overall orientation of the cargo NLSs different (e.g., in SREBP, the a-helices are bound perpendicular to Imp $\beta 1$, whereas the IBB domains bind parallel with the $\operatorname{Imp} \beta 1$ superhelix) but also the overall helicoidal twist of $I m p \beta 1$ is different, with IBB domain-bound Imp $\beta 1$ forming a more closed conformation.

\section{Imp $\beta$ 1:Ran interactions}

Dissociation of the nuclear import cargo from Imp $\beta 1$ following entry into the nucleus is mediated by RanGTP binding [5,8-10]. The structures for truncated human Imp $\beta 1$ [27] and full-length ylmp $\beta 1$ [28] in complex with RanGTP (Fig. 2) have been determined, revealing important allosteric mechanisms of cargo-release control. The $\mathrm{N}$-terminal fragment of Imp $\beta 1$, encompassing HEAT repeats 110 (residues 1-462) bound to RanGTP, identified two main contact areas: (1) HEAT repeat 1 interacting with Ran switch-II region residues $W^{64}, G^{74}$ and $Q^{82}$; HEAT repeat 2 interacting with Ran residues $E^{70}$, $\mathrm{D}^{77} \mathrm{G}^{78}, \mathrm{~N}^{103}$ and $\mathrm{D}^{107}$; HEAT repeat 3 interacting with Ran $\mathrm{R}^{110}, \mathrm{~V}^{111}$ and $\mathrm{E}^{113}$; and (2) HEAT repeat 7 and the highly conserved acidic loop in HEAT repeat 8 interacting with Ran residues $\mathrm{R}^{140} \mathrm{KKNLQYY}, \mathrm{K}^{159}$, $R^{166}$ and $P^{172} N^{173}$. The structure of the complex with the full-length yeast orthologue (Kap95p; ylmp $\beta 1$ ) 
revealed an additional interaction site within RanGTP, involving the Ran switch-I loop and the C-terminal arch of ylmp $\beta 1$, producing a change in curvature that locks ylmp $\beta 1$ in a conformation incompatible with cargo binding. A sequential binding mechanism has been proposed to occur for RanGTP binding at three distinct sites, with residues in the switch-II loop binding to the CRIME motif in N-terminal HEAT repeats 1-4 first, followed by the basic patch on Ran $\left(\mathrm{K}^{134}\right.$, $\mathrm{H}^{139} \mathrm{RK}$ ) binding to acidic residues in HEAT repeats 7 and 8 and finally the switch-I loop binding HEAT repeats $12-15$ residues $R^{29} ; K^{37}$ forming salt bridges and $\mathrm{H}$-bonds; $\mathrm{F}^{35}$ hydrophobic interactions; and $\mathrm{K}^{152}, \mathrm{~N}^{154}, \mathrm{~N}^{156}$ and $\mathrm{F}^{127}$ also contributing to the interface. The third site, where the RanGTP switchI loop binds the C-terminal arch of ylmp $\beta 1$, is crucial for locking the molecule in a conformation with increased curvature that cannot bind cargo. This allosteric mechanism enables the release of cargo because it results in Imp $\beta 1$ becoming locked in a conformation that prevents the flexibility required to bind to different partners. The size of the ylmp $\beta 1$ :RanGTP interface $\left(2159 \AA^{2}\right.$ [28]) is similar to the interfaces found in cargo and adaptor complexes, and there is limited overlap between the binding sites. The data therefore strongly support an allosteric mechanism of control for cargo release.

The structure of the ylmp $\beta 1$ :RanGDP complex revealed further insights into Ran binding in the nucleus and cytoplasm [29]. The crystal structure showed that the Ran switch-I and switch-II regions are induced by ylmp $\beta 1$ into a GTP-bound conformation so that, rather than these switch regions precluding binding to $y \operatorname{lmp} \beta 1$, they are forced into conformations that enable ylmp $\beta 1$ binding. This is not inconsistent with other reports of binding partner-induced conformational changes within Ran and Ras switch regions [27,30]. One region that was shown to be differentially positioned in ylmp $\beta 1: R a n G D P$ and $y / m p \beta 1: R a n G T P$ structures corresponds to the "basic loop" contained with Ran, comprising residues 133-144, which move $8 \AA$ between the two structures. Significantly, this structure highlights that the ylmp $\beta 1$ HEAT repeats 7 and 8 are exposed in the RanGDP complex, allowing binding partners such as Impa to disassemble the complex [29].

\section{Impß1 and RanGTP dissociation and recycling}

After translocation to the cytoplasm, recycling of $\operatorname{Imp} \beta 1$ and other transport factors is achieved by the conversion of Ran into the GDP-bound state. Accessory proteins located at the cytoplasmic face of the NPC facilitate the dissociation of the kinetically stable importin:RanGTP complexes and the generation of RanGDP; the intrinsic GTPase activity of Ran is low and GTP hydrolysis is prevented when Ran is in complex with $\beta$-Kap family members [31]. Although the precise mechanism of RanGTP dissociation from transport factors is unclear, one model implicates RanGAP, together with Ran-binding domain RanBDs from either Ran binding protein RanBP1 or RanBP2 [31]. The structure of Ran in complex with RanBD1 (from RanBP2) and a fragment of Imp $\beta 1$ (residues 1-462) has been determined [32] (Fig. 3A), and superimposing it on the Imp $\beta 1$ :RanGTP complex identifies clashes between the Imp $\beta 1$ C-terminal HEAT repeats and RanBD1. This suggests that the primary role of RanBDs is to destabilize the interaction between the transport factors and RanGTP through steric hindrance.

The structure of Ran:GppNHp (a non-hydrolysable GTP analogue) in complex with RanBD1 (of RanBP2) and RanGAP has also been determined [33] (Fig. 3B), and comparison with the Imp $\beta 1$ :RanGTP complex reveals further clashes between Imp $\beta 1$ and RanGAP. In vitro radiolabeled-nucleotide assays have demonstrated that RanBP1 does not affect the intrinsic GTPase activity of Ran in isolation. However, RanBP1
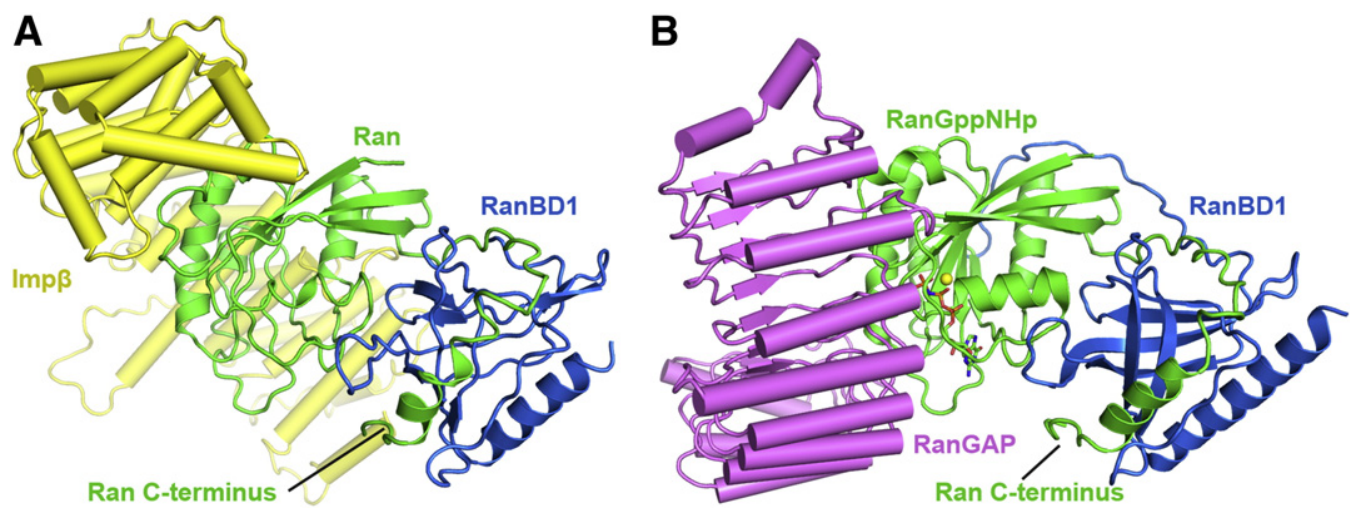

Fig. 3. Structures of (A) Ran:RanBD1:Impß1(1-462) [32] and (B) RanGppNHp:RanBD1:RanGAP complexes (PDB entry 1K5D). RanBD1 forms a molecular embrace with Ran, sequestering Ran's C-terminus. Superimposition of the full-length RanGTP-Imp $\beta$ structure onto the ternary complexes reveals steric clashes between Imp $\beta$ and RanBD1 or RanGAP. 
has been shown to co-stimulate, with RanGAP, GTP hydrolysis by Ran [34], as well as increase the association rate of RanGTP and RanGAP [35]. This is consistent with the crystal structures of the ternary complexes; RanBP1 forms a molecular embrace with Ran, sequestering Ran's C-terminal region that inhibits RanGAP-mediated GTP hydrolysis. Moreover, the structure of the ternary complex indicated that, unlike other Ras-family GAPs, RanGAP does not provide catalytic residues to stimulate RanGTP hydrolysis $[36,37]$. Instead, Ran contains all requisite catalytic machinery and the primary role of RanGAP appears to be the stabilization of the Ran switch-II loop and the repositioning of Ran's catalytic $Q^{69}$ residue toward the active site [33].

In contrast to its GTP-bound state, RanGDP has low affinity for transport factors $(\sim 2 \mu \mathrm{M}$ for Imp $\beta 1)$ [29], RanBDs $(\sim 10 \mu \mathrm{M})[38,39]$ and RanGAP $(\sim 100 \mu \mathrm{M})$ [35]. GTP hydrolysis therefore precludes rebinding of cytoplasmic RanGDP to transport factors or accessory proteins, rendering dissociation essentially irreversible on the one hand while enabling the recycling of its binding partners for further rounds of import and disassembly on the other.

\section{Imp $\beta 1$ :Nup interactions}

To mediate translocation across the nuclear envelope, Imp $\beta 1$ interacts directly with Nups that contain tandem repeats of motifs based on a Phe-Gly core (FG Nups). The structures of Nup FxFG and GLFG motifs bound to ylmp $\beta 1$ and $I m p \beta 1$ show that the interaction sites are primarily located on the convex outer surface in pockets between the A-helices (Fig. 2). HEAT repeats 5 and 6 bind both the GLFG and FxFG peptides $[40,41]$. The highaffinity Nup1p (residues 963-1076) binding sites on ylmp $\beta 1$ are located between the A-helices of HEAT repeats 5-8. The first and second Nup1p (residues 974-988) binding sites are between HEAT repeats 7 and 8 and HEAT repeats 6 and 7 , respectively, with the third site between HEAT repeats 5 and 6 . Because of the repetitive sequences within the Nup1p C-terminal domain, it is unclear whether the binding involves Nup1p residues 999-1011 or residues 1019-1031. The interactions within all three sites are predominantly hydrophobic and involve the Phe aromatic rings, as well as contributions from the adjacent hydrophobic residues (site 1, $\mathrm{F}^{977}$ and $\mathrm{P}^{979}$; site $2, \mathrm{~F}^{987}$ and $\mathrm{I}^{985}$; site $3, \mathrm{~F}^{1008}$ and $\mathrm{I}^{1007}$ or $\mathrm{F}^{1027}$ and $\left.\mathrm{I}^{1026}\right)$. There is also an additional hydrophobic interaction between $\mathrm{P}^{983}$ and HEAT repeat 7 , as well as several $\mathrm{H}$-bonds to the peptide backbone of Nup1p at each site. The total buried surface area of interactions between $\operatorname{Imp} \beta 1$ and other FG Nup cores is $\sim 1000 \AA^{2}$. However, for the ylmpß1:Nup1p FxFG, the interaction of the buried surface area of the three binding sites is twice that observed for the other interactions, with $2210 \AA^{2}$ buried [41]. Although the higher affinity of Nup1p for ylmp $\beta 1$ cannot offer a mechanism for translocation, concentrating ylmp $\beta 1$ at the nuclear face can enhance the kinetics of import complex dissociation and thus the overall rate of transport [41].

Bednenko et al. proposed that there was a second, weaker, FG binding site on human Imp $\beta 1$, located between HEAT repeats 14 and 16 and that involved Leu ${ }^{612}$, $\mathrm{Phe}^{688}$ and Leu ${ }^{695}$ [42]. Although mutations of this site alone did not impair the binding of a FG peptide, these mutations did impair function in conjunction with mutations in the primary FG binding site. Molecular dynamics calculations [43] indicated that there may be additional FG binding sites on Imp $\beta 1$, but this work has not been validated by mutagenesis.

\section{Transportin-1/PY-NLS-mediated nuclear import}

Transportin-1 (Trn1) imports a broad spectrum of cargoes, many of which are mRNA-binding proteins or transcription factors. Like other $\beta$-Kaps, Trn1 is composed of a series of HEAT repeats arranged as C- and N-terminal arches (reviewed in Ref. [16]). Compared to Imp $\beta 1$, it contains one additional HEAT repeat and a large 62-residue loop that connects the two helices within HEAT repeat 8 and that appears to be involved in cargo release upon RanGTP binding. Trn1 binds cargoes directly, recognizing them through a broad range of loosely related NLSs that have a characteristic C-terminal Pro-Tyr (PY) motif, which generally has an Arg preceding it by $2-5$ residues, giving a consensus of $\mathrm{RX}_{2-5} \mathrm{PY}$ [44]. The motif in the best-characterized cargo, the splicing factor hnRNP A1, is known as the M9-NLS [45-47]. These motifs lack defined elements of secondary structure so that they can adopt a conformation to match the binding surface on Trn1. These NLSs generally also contain either a basic or a hydrophobic cluster N-terminal to the PY motif. The hydrophobic PY-NLSs contain two motifs separated by 8-13 amino acids, an N-terminal $\Phi-G / A / S U$ motif ( $\Phi$ represents a hydrophobic amino acid) and a C-terminal sequence $\mathrm{R} / \mathrm{K} / \mathrm{H}-\mathrm{X}_{2-5}-\mathrm{P}-\mathrm{Y}$. Structures of a range of these NLSs bound to Trn1 show considerable variability in the way they bind. The region in the C-terminal arch of Trn1, to which the $\mathrm{N}$-terminal clusters in the NLSs bind, is rich in negatively charged resides and also contains a number of scattered clusters of hydrophobic residues, enabling it to accommodate a considerable range of different NLS sequences [48,49]. Structure-guided mutations have not always been successful in disrupting these interactions, reflecting the complexity with which Trn1 recognizes its cargoes. Like other $\beta$-Kaps, Trn1 binds its cargoes in the cytoplasm, where Ran is primarily in its GDP-bound 
state and releases it in the nucleus when RanGTP binds.

\section{Trn1:hydrophobic PY-NLS complexes}

The available structures include the complexes with the NLSs from the heterogeneous nuclear ribonucleoproteins hnRNP $A 1$ and $D$, nuclear RNA export factor TAP, hnRNP D-like protein JKTBP and fused in sarcoma protein (FUS) [44,50,51] (Fig. 4). In the Trn1:hnRNP A1 crystal structure, the so-called M9-NLS (residues 257-305) binds the C-terminal arch of Trn1, comprising HEAT repeats 8-20. The hydrophobic $\mathrm{N}$-terminal motif residues $\mathrm{F}^{273}$ and $\mathrm{P}^{275}$ form hydrophobic contacts with Trn1 residues $I^{773}$ and $W^{730}$, respectively. Beyond $P^{288} Y^{289}$, the PY-NLS is disordered. Residues 263-266 in hnRNP A1 interact with a hydrophobic patch in HEAT repeats 18 and 19 on the outer convex surface, as well as with HEAT 20 on the inner concave surface. Residues 267-269 bind the loop in HEAT repeat 18, while the rest of hnRNP A1 follows the inner concave C-terminal arch to contact HEAT repeats 8-17. Isothermal calorimetry analysis of site-directed mutants indicates that the most significant energy contributions to the interaction come from the N-terminal hydrophobic motif [48]. The hnRNP A1 M9-NLS is antiparallel with the Trn1 superhelix, and the interaction buries $3432 \AA^{2}$ of surface area.

Trn1 cargo binding involving other hydrophobic PY-NLSs follows an analogous pattern, with the hydrophobic hnRNP D PY-NLS (residues 332-355) binding to Trn1 HEAT repeats 8-18, whereas the TAP NLS (residues 53-82) binds to HEAT repeats 8-13 (but not HEAT repeats 14-18); the JKTBP NLS (residues 396-420) binds to HEAT repeats 8-13.

Similar to other PY-NLSs, FUS PY-NLS (residues 498-526) occupies the C-terminal arch of Trn1, but unlike other PY-NLSs that are structurally disordered, the central segment of the FUS peptide (residues 514-522) forms a 2.5-turn $\alpha$-helix. The FUS PY-NLS interacts with Trn1 at three major sites. The first site involves the $\mathrm{N}$-terminal residues 508-511 of FUS forming hydrophobic interactions with Trn1 residues $\mathrm{W}^{730}$ and $\mathrm{I}^{773}$. Within this $\mathrm{N}$-terminal
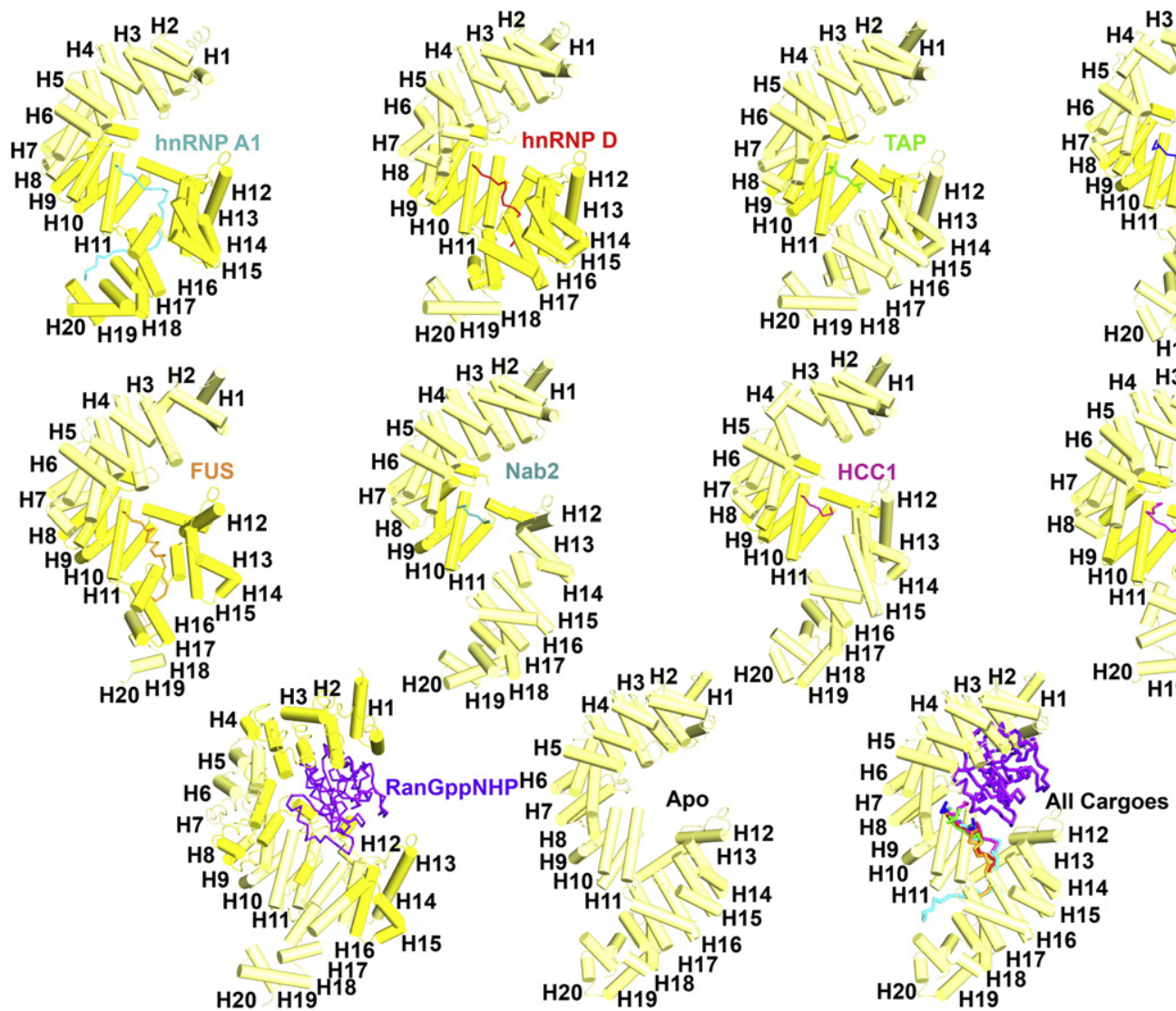

Fig. 4. Structures of Trn1. PDB entries: hnRNP A1 complex (2H4M), hnRNP D complex (2Z5N), TAP complex (2Z5K), JKTBP complex (2Z5O), FUS complex (4FQ3), Nab2 complex (4JLQ), HCC1 complex (4OO6), hnRNP M complex (2OT8), RanGppNHP complex (1QBK) and apo (2Z5J). The HEAT repeats involved in cargo binding are highlighted in dark yellow. A representative Trn1 in apo-form has all cargoes overlaid. 
hydrophobic motif, FUS $\mathrm{K}^{510}$ makes hydrophobic interactions with Trn1 residues $W^{730}$ and salt bridges with Trn1 $E^{653}$ and $D^{693}$. In the second "central" site, FUS residues 514-522, arranged as an a-helix, interact with Trn1 HEAT repeats 9-12 at residues $D^{509}, D^{543}, D^{550}, E^{588}$ and $D^{646}$, forming $\mathrm{H}$-bonds and salt-bridge interactions with all five of its basic residues $\left(R^{514}, H^{517}, R^{518}, R^{521}\right.$ and $\left.R^{522}\right)$. In the third site, the C-terminal residues $P^{525} Y^{526}$ interact primarily hydrophobically with $\operatorname{Trn} 1$ residues $A^{381}$, $L^{419}, I^{457}$ and $W^{640}$. Overall, the FUS PY-NLS binds Trn1 through hydrophobic interactions at both the $\mathrm{N}$-terminus and the C-terminus of the peptide and through electrostatic interactions in its central $\alpha$-helix. Recently, FUS binding to Trn1 was shown to also occur within the adjacent unmethylated RGG3 domain (residues 472-507), suggesting that it could act independently as a Trn1-dependent NLS or as an accessory domain capable of extending the PY-NLS [52].

Overall, the C-terminal PY motif and the $\mathrm{N}$-terminal hydrophobic motif in these PY-NLSs are recognized by Trn1 HEAT repeats 8-13 and HEAT repeats 1418 , respectively.

\section{Trn1:basic PY-NLS complexes}

In the structure of Trn1 in complex with yNab2 (residues 205-242) [51] (Fig. 4), Nab2 residues $\mathrm{T}^{234}{ }^{2} \mathrm{R} N \mathrm{LL}^{240}$ bind Trn1 in an extended conformation, occupying the same binding site observed by the $\mathrm{RX}_{2-5} \mathrm{PY}$ motifs in other Trn1:PY-NLS structures. The Nab2 PY-NLS structure contains a "PL" instead of the canonical "PY". The N-terminal $R^{235}$ makes salt-bridge and $\mathrm{H}$-bond interactions with $D^{543}, T^{506}$, $\mathrm{E}^{509}$ and $\mathrm{T}^{547}$ of Trn1. Additionally, $\mathrm{F}^{236}$ makes hydrophobic contacts with Trn1 residues $A^{499}, E^{498}$ and $\mathrm{W}^{460} ; \mathrm{P}^{238}$ interacts predominantly through hydrophobic interactions with Trn1 residues $L^{419}$, $\mathrm{I}^{457}$ and $\mathrm{W}^{460}$; and $\mathrm{L}^{239}$ interacts hydrophobically with Trn1 residues $L^{419}, A^{381}, A^{422}$ and $W^{460}$. The Tyr aromatic ring can make more hydrophobic or polar interactions, compared to the Leu in the PL motif, resulting in a higher energetic contribution.

The structure of the Trn1:HCC1 (hepatocellular carcinoma protein 1) complex (PDB ID 40O6) has been deposited; however, a detailed description of the structure has not yet been published. We provide a brief comparison with other similar NLSs. HCC1 contains a basic PY-NLS and analysis of the interface with Trn1 reveals residues $R^{92}$ GRYRSPY ${ }^{99}$ that bind HEAT repeats 8-14, with $\mathrm{H}$-bonds formed between HCC1 $Y^{95}, R^{96}$ and $Y^{99}$, as well as Trn1 residues $D^{384}, A^{423}, S^{502}$ and $T^{506}$.

The basic PY-NLS of hnRNP M binds Trn1 HEAT repeats 8-16 [48]. Unlike hnRNP A1 that binds the convex side of the $\mathrm{N}$-terminal Trn1, the $\mathrm{N}$-terminus of hnRNP $\mathrm{M}$ binds toward the Trn1 arch opening. In the $\mathrm{N}$-terminal basic motif of hnRNP $\mathrm{M}$, residues
$\mathrm{E}^{51} \mathrm{KNI}^{54}$ bind the same region of Trn1 as hnRNP A1 residues 274-277. However, in contrast to hnRNP A1, hnRNP $M$ is ordered beyond the PY motif with five residues extending C-terminally. Hydrophobic interactions involve aliphatic portions of hnRNP M residues $\mathrm{K}^{52}$ and $\mathrm{I}^{54}$ and Trn1 residues $\mathrm{W}^{730}, \mathrm{I}^{642}, \mathrm{D}^{646}$ and $\mathrm{Q}^{685}$. Surprisingly, unlike hnRNP $A 1$, the most significant energy contributions come from the $\mathrm{C}$-terminal $\mathrm{PY}$ domain rather than from the $\mathrm{N}$-terminal motif.

Overall, although Trn1 uses the same residues (HEAT repeats 8-13) to bind the C-terminal PY-NLS motifs in all PY-NLSs, the $\mathrm{N}$-terminal regions of basic and hydrophobic PY-NLSs only partially overlap.

\section{Trn1:Ran complex}

Similar to other $\beta$-Kap family members, Ran binding to Trn1 in the nucleus displaces the imported cargo. Trn1 has a characteristic acidic H8 loop, and the proposed NLS dissociation mechanism involves RanGTP binding to the Trn1 N-terminal arch. This causes conformational changes that push the $\mathrm{H} 8$ loop into the principal cargo-binding site in the $\mathrm{C}$-terminal arch of Trn1, causing cargo release [53]. The structure of Trn1:RanGTP complex (Fig. 4) [54] reveals two distinct binding interfaces: an N-terminal interface involving interactions between Trn1 HEAT repeats 1-4 and the switch regions of Ran (residues 64-110) and a more centrally located interface involving HEAT repeats 7-8 and 14-15 and loop-8 (residues 311$373)$ of Trn1 binding to Ran $\alpha$-helices $\alpha 4$ and $\alpha 5$, $\beta$-strand $\beta 6$ and the intervening loops. In the N-terminal interface, the C-terminal region of Ran switch-I (residues 44-47) interacts with Trn1 HEAT repeat 1; the switch-II region of Ran (residues 72-82) is buried at the interface by hydrophobic contacts to Trn1 HEAT repeat 2. Overall, these interactions mediate a combined buried surface area of $3900 \AA^{2}$ and involve both polar and hydrophobic contacts, with most of the polar contacts contributed by Ran $R^{106}$ and $R^{110}$ and Trn1 $\mathrm{S}^{165}, \mathrm{D}^{164}$ and $\mathrm{E}^{161}$.

\section{Transportin-3/RS repeat NLS-mediated nuclear import}

Transportin-3 (Trn3), composed of 20 HEAT repeats, mediates the nuclear import of many proteins containing arginine-serine ("RS") repeat NLSs [55-57]. These proteins are typically involved in mRNA metabolism and include the alternative splicing factor/splicing factor ASF/SF2. Structures of Trn3 bound to ASF/SF2 and Ran, and its unbound form, are available [58] (Fig. 5). The flexibility of the HEAT repeat region is important for binding of the $\mathrm{RS}$ domains, as well as the RNA recognition motif in ASF/SF2. The significant overlap in binding regions between RanGTP and the cargo explains the structural basis of release. 


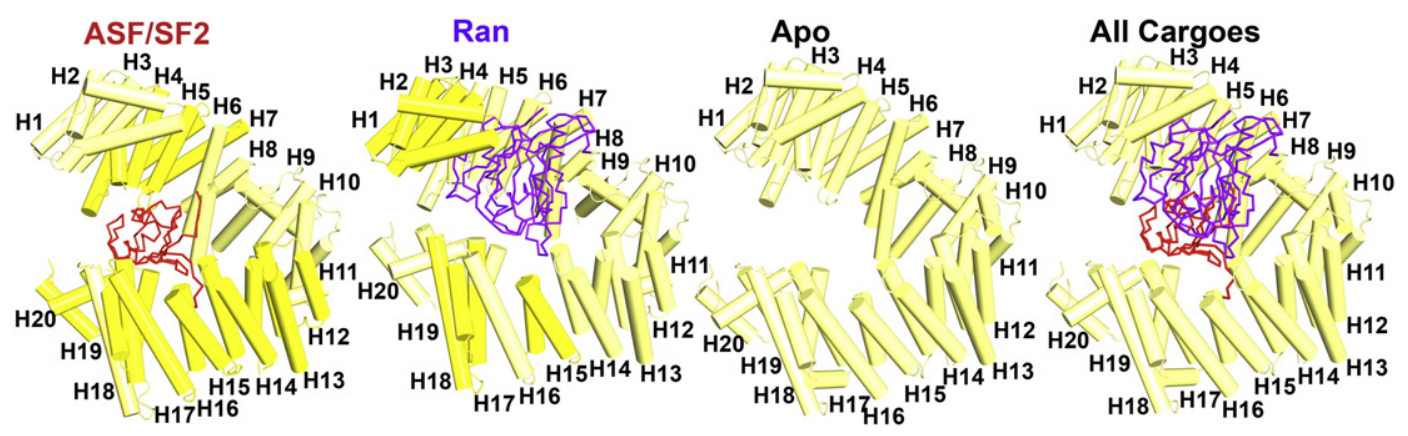

Fig. 5. Structures of Trn3. PDB entries: ASF/SF2 complex (4C0O), Ran complex (4COQ) and apo (4COP). The HEAT repeats involved in cargo binding are highlighted in dark yellow. A representative Trn3 in apo-form has all cargoes overlaid.

\section{Trn3:ASF/SF2 cargo complex}

The ASF/SF2 protein is an RNA-splicing factor that contains two RRM domains and an RS domain. Residues within HEAT repeat 15, particularly Arg-rich regions within B-helices, are the key binding determinants for the recognition of the phosphorylated RS domain of ASF/SF2 [58]. There are three major binding regions, which bury a total of $\sim 4300 \mathrm{~A}^{2}$ of surface area. The RRM domain (residues 116-191) binds HEAT repeats 4-7 and 19-20, the RS regions (residues 198-211) are bound by HEAT repeat domains 14-17 and the linker region between the RRM and RS domains is bound by HEAT repeat domains 12 and 13 [58]. These interactions are mediated by an extensive array of salt bridges. Although the RRM domain exhibited $60 \%$ of the buried surface area, the RS domain is actually the major contributor to binding; this is based on (i) mutagenesis studies, (ii) the fact that many RS domain-containing proteins that interact with Trn3 do not contain RRM domains and (iii) the RS-domain NLS being necessary and sufficient to mediate nuclear transport [58].

\section{Trn3:Ran complex}

Similar to other $\beta$-Kap family members bound to Ran, the structure of Trn3 in complex with RanGTP shows that Ran contacts the B-helices on the concave side of the transport receptor. Sites that mediate binding within Ran include the switch-I and switch-II regions, which interact with the Trn3 HEAT repeats $1-3$ and HEAT repeats 17 and 18 , respectively [58]. In particular, the switch-I region of Ran inhibits the ability of HEAT repeat 15 to interact with the RS domain.

\section{Apo-Trn3}

The 20 HEAT repeats in apo-Trn3 are arranged in a circular shape, whereby the $\mathrm{N}$ - and $\mathrm{C}$-terminal repeats face each other. In most $\beta$-Kap family members, HEAT repeats pack in a rather uniform manner, but in Trn3, there are several notable exceptions: HEAT repeats 1 and 2 pack perpendicular to each other, the stacking of HEAT repeats $3 / 4$ and $9 / 10$ displays pronounced left-handed twists and HEAT repeat 20 contains an additional C-terminal $\alpha$-helix. Interestingly, the crystal structure revealed a significant molecular interface mediating homodimer formation, and small-angle X-ray scattering analysis is consistent with this observation; however, the functional role of dimerization is unclear at this stage [58].

\section{Importin-13-mediated nuclear transport}

Importin-13 (Imp13) is the closest paralogue of Trn3 but has distinctly different cargo recognition specificity [59]. Whereas Trn3 predominantly interacts with flexible RS domains, Imp13 mediates the nuclear import of several transcription factors containing histone-fold motifs (composed of $\sim 70$ amino acids arranged as three $\alpha$-helices). Similar to Trn1, Imp13 contains 20 consecutive HEAT repeats [60] and interactions with cargo occur on the inner concave surface; however, Imp13 is able to mediate transport of cargoes both into and out of the nucleus. Recent co-crystal structures of Imp13 with the exon junction complex components Mago and $\mathrm{Y} 14$, as well as the E2 SUMO-conjugating enzyme UBC9, show that the flexibility of Imp13 is important for cargo binding (Fig. 6).

\section{Imp13:Mago-Y14 cargo complex}

The first structure of Imp13 corresponds to the complex with Mago-Y14, revealing that 15 HEAT repeats are involved in binding the cargo [60]. Imp13 adopts a closed ring-like conformation, whereby the $\mathrm{N}$ - and $\mathrm{C}$-terminal arches are facing each other, and Mago-Y14 binds to the inner concave-surface helices of the C-terminal arch. HEAT repeats 8 and 9 interact with the Mago $\beta$-sheet, at the site where the $\mathrm{N}$ terminal region of $\mathrm{Y} 14$ is bound, and HEAT repeat 


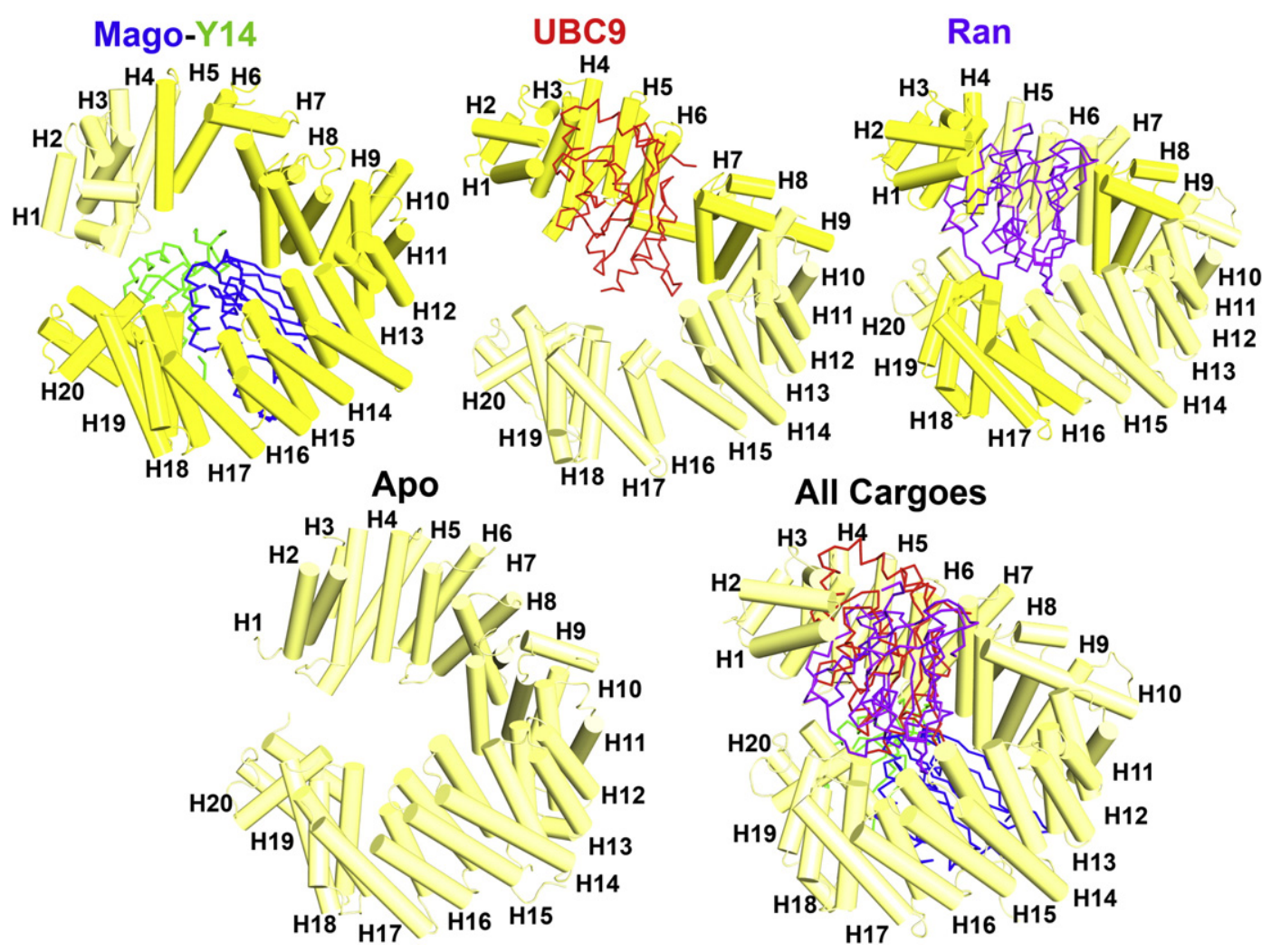

Fig. 6. Structures of Imp13. PDB entries: Mago-Y14 complex (2X1G), UBC9 complex (2XWU), Ran complex (2X19) and apo (3ZKV). The HEAT repeats involved in cargo binding are highlighted in dark yellow. A representative Imp13 from Imp13:Ran complex has all cargoes overlaid.

15 binds at the opposite side of the Mago $\beta$-sheet. HEAT repeats 17, 18 and 20 interact with Mago a-helices, and HEAT repeats 4-7 and HEAT repeats 19 and 20 surround $\mathrm{Y} 14$.

\section{Imp13:UBC9 cargo complex}

The crystal structure of the Imp13:UBC9 cargo complex showed a unique cargo recognition mode, with UBC9 bound within the N-terminal arch of Imp13 to occupy the RanGTP-binding site in that region [61]. Unlike the Imp13:Mago-Y14 complex, the $\mathrm{N}$ - and C-terminal HEAT repeats of Imp13 are positioned away from each other. UBC9 lies between HEAT repeats 1 and 9 and forms interactions within the inner concave surface of Imp13. UBC9 makes interactions mainly through three of its loops. The first interacting region involves hydrophobic interactions mediated by a loop and a helix that bind both helices within HEAT repeat 1 and the B-helix of HEAT repeat 2 of Imp13. $\mathrm{I}^{125}$ of UBC9 makes contact with Imp13 residues $Y^{34}$, $\mathrm{E}^{73}$ and $\mathrm{Y}^{76}$, while an additional hydrophobic interaction involves UBC9, $Y^{134}$, positioned toward Imp13 $\mathrm{L}^{33}$ and $\mathrm{Y}^{34}$.

\section{Imp13:Ran complex}

Similar to $\operatorname{Imp} \beta 1$ and Trn1, cargo release and directionality of nuclear import of Imp13 are achieved by Ran. However, cargo release of Imp $\beta 1$ and Trn1 relies on the characteristic acidic loop within HEAT repeat 8 , which is lacking in Imp13, and therefore, the mechanism of cargo release is likely to be different in these transport molecules. The Imp13:RanGTP structure shows RanGTP interacting with the inner concave helices contained within the N-terminal arch of Imp13 at 3 sites, similar in position to those identified in Imp $\beta 1$, Trn1 and Trn3 [62]. The Ran switch-I loop binds Imp13 at HEAT repeats 16-19 with predominantly polar and electrostatic contacts (e.g., Ran $\mathrm{K}^{39} \mathrm{~K}^{40}$ binding Imp13 $\left.D^{785} / D^{788}\right)$ [62]. The Ran switch-II loop binds Imp13 HEAT repeats $1-3$, with hydrophobic interactions involving Ran $\mathrm{L}^{77}$ and electrostatic contacts between Ran $D^{79}$ and Imp13 $R^{122}$. The helix adjacent to the switch-II loop also contacts HEAT repeats 3 and 4; in particular, Ran residues $R^{108}$ and $R^{112}$ contact Imp13 residues $\mathrm{E}^{175}$ and $\mathrm{E}^{176}$ [62]. The third binding site of RanGTP is through Imp13 HEAT repeats 8 and 9 , with Ran residues $R^{168} K^{169}$ 
approaching negatively charged Imp13 residues $D^{415} E^{416}$ on helix 9B. Thus, unlike the mechanism of cargo release by $\operatorname{Imp} \beta 1$, Imp13 releases cargo due to its direct steric clashes with RanGTP.

\section{Impa-Mediated Nuclear Import}

In this pathway, the Impa:Imp $\beta 1$ heterodimer binds to cargo proteins containing cNLSs [63]. The translocation through the nuclear pore is achieved through transient interactions between Imp $\beta 1$ and Nups. This process, known as the classical nuclear import pathway, is thought to be the most extensively used nuclear import mechanism in the cell [64-66].

\section{Monopartite and bipartite cNLSs}

The first nuclear targeting motif was identified in the simian virus SV40 large T-antigen (TAg) through mutational studies. It comprises a small stretch of positively charged amino acid residues $\left(P^{126} K_{K K R R V}{ }^{132}\right)$. Non-conservative substitutions of residues within this motif abrogated nuclear distribution of the cargo protein [67]. Furthermore, fusion of this motif to cytoplasmic proteins such as $\beta$-galactosidase induced their nuclear accumulation [68]. A similar, but more complex, signal was later defined for the Xenopus laevis nucleoplasmin protein, consisting of two clusters of basic amino acids separated by a 10- to 12-residue linker region (K ${ }^{155}$ RPAATKKAGQAKKKKK ${ }^{170}$ ) [69]. Substitution of residues within either basic cluster altered the nuclear distribution of the protein, suggesting that both motifs were required for nuclear targeting. By contrast, mutation of residues within the linker region had no effect on nuclear distribution [70].

The two sequences are now commonly described as the prototypic monopartite (SV40-TAg) and bipartite (nucleoplasmin) cNLSs and numerous cNLS-containing cargo proteins have since been identified based on sequence similarity with these two [65] (Supplementary Table 2). Using in vitro transport assays in digitonin-permeabilized cells, we showed the active import of cNLS sequences to be dependent on soluble cytoplasmic factors [71]. This in vitro system was later used to identify and characterize essential transport factors, Impa and Imp $\beta 1$, that could reconstitute nuclear import of cNLS sequences when reintroduced to cytosol-depleted cells [63,72-76]. Consequently, cNLS cargoes are defined by the presence of one or two sequence clusters rich in Arg and Lys that are necessary and sufficient for nuclear import by the Impa:Imp $\beta 1$ complex.

\section{Structure of Impa}

Impa has a modular structure composed of a short $\mathrm{N}$-terminal auto-inhibitory region that also mediates binding to Imp $\beta 1$ [77] (the IBB domain) and larger C-terminal NLS binding domain composed of 10 ARM repeats $[78,79]$. The ARM repeat motif, first described for the Drosophila melanogaster armadillo protein [80], is composed of three a-helices $(\mathrm{H} 1, \mathrm{H} 2$ and $\mathrm{H} 3$ ). The continuous stacking of the tandem ARM repeats generates a superhelical solenoid, with the H3 helices forming the Impa inner concave surface (Fig. 7A). A rotation between consecutive ARM repeats creates a groove along the superhelical axis of the protein, where the NLS binding sites are located [11]. The ARM repeat solenoid appears to be much less flexible than the HEAT repeat solenoids in $\beta$-Kaps. The structures of Impa proteins from different organisms have been determined (human [81-85], Saccharomyces cerevisiae $[13,79,86]$, mouse [12,87-102], rice [100,103], Arabidopsis thaliana [104] and Neurospora crassa [105]; Supplementary Table 3). These structures all comprise 10 ARM repeats, but their curvatures vary, particularly between proteins from different phylogenetic families [128]. The structural variations result in differences in binding NLSs, as observed for rice and mouse Impa bound to the same NLS peptide, for example [66].

\section{Structural basis of cNLS recognition by Impa}

X-ray crystallography has been used extensively to elucidate the molecular details of cNLS binding to Impa. The concave surface formed by Impa H3 helices comprises the cNLS binding site, which displays a high degree of sequence conservation between Impa proteins from various organisms (Fig. 7B). More specifically, conserved $(\wedge \mathrm{R} / \mathrm{K}) \mathrm{XXWXXXN \text {motifs }}$ (where $x$ is any residue, and $\wedge R / K$ is any residue other than Arg/Lys, typically an acidic or hydrophilic residue) within the $\mathrm{H} 3$ helices form an array of binding pockets along the inner concave groove of the Impa adaptor. The conserved Asn residues form $\mathrm{H}$-bonds with the cNLS backbone, whereas the invariant Trp side chains form an array of binding cavities on the adaptor surface, typically with acidic residues $\left({ }^{\wedge} \mathrm{R} / \mathrm{K}\right)$ located at the end of these pockets. Thus, the aliphatic moieties of long basic side chains, such as Lys and Arg, can interact with the stacked indole rings of the Trp array, while the positively charged portion of the side chain can simultaneously form $\mathrm{H}$-bonds and salt bridges with the hydrophilic residues that line the pockets and can also form cation- $\Pi$ interactions with the electron clouds of the tryptophan indoles.

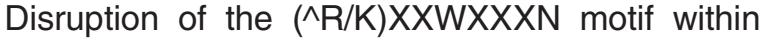
ARM repeats 5 and 6 has been observed in all $I \mathrm{mpa}$ proteins with known structure. This disruption effectively creates and segregates two distinct binding 
A

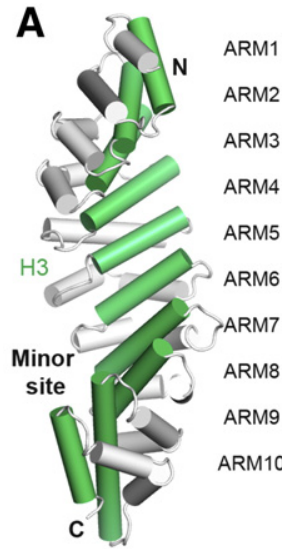

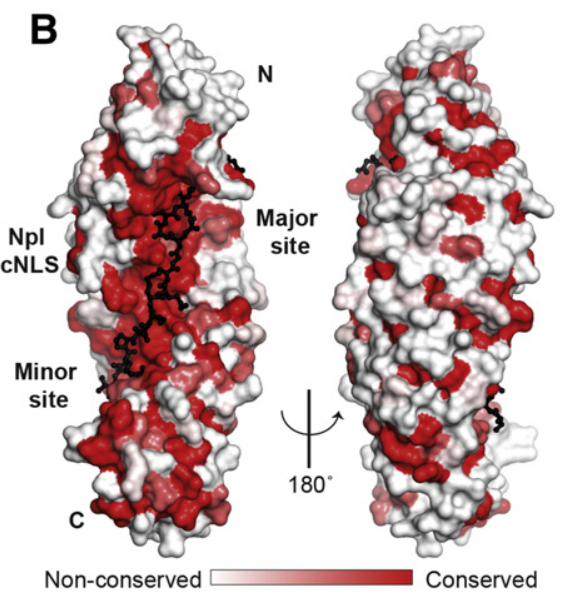

C

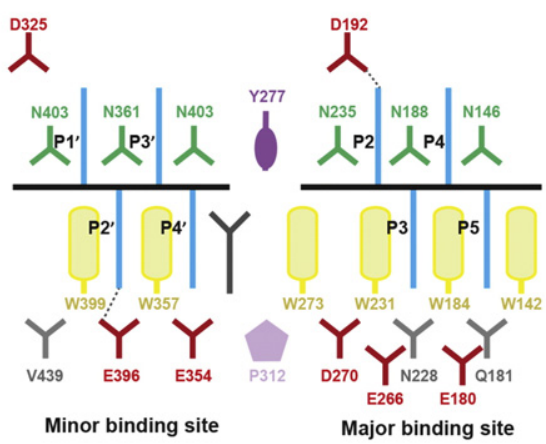

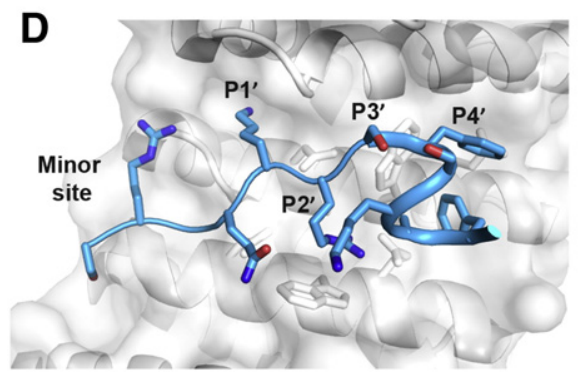

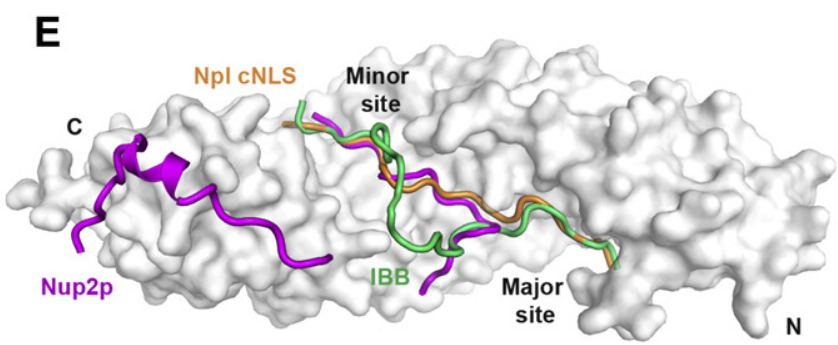

Fig. 7. NLS binding by Impa. (A) Structure of rice Impa (PDB entry 4BQK) with $\mathrm{H} 3$ helices colored green. (B) Structure of mouse Impa (PDB entry 3UL1) in complex with nucleoplasmin (Npl) cNLS (shown as black sticks). The mouse adaptor is colored by sequence conservation based on known Impa structures (human Impa1, human Impa3, human Impa5, human Impa7, Mus musculus Impa1, S. cerevisiae Impa, Oryza sativa Impa, A. thaliana Impa3 and N. crassa Impa1). (C) Schematic representation of a monopartite NLS binding at the Impa major and minor site pockets. Conserved Asn and Trp residues of Impa shown in green and yellow, respectively. Monopartite NLS main chains and side chains are shown as black and blue lines, respectively. Broken lines indicate common salt-bridge interactions at the P2- and P2'-binding cavities. (D) Structure of mouse Impa with atypical minor site-binding Gua NLS shown in blue. Impa residues comprising

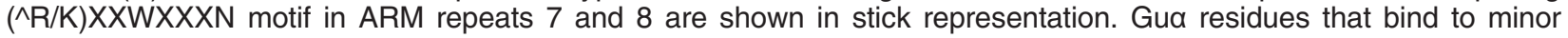
site cavities are indicated. (E) Structure of full-length yeast Impa (PDB entry 1WA5; IBB domain shown in green) superimposed onto yeast Impa:Nup2p (PDB entry 2C1T; Nup2p shown in magenta) and yeast Impa:nucleoplasmin cNLS (PDB entry 1EE5; NLS shown in orange) complexes. For clarity, only one Impa ARM repeat domain is shown in gray surface representation.

regions on the Impa surface termed the major (ARM repeats 2-4) and the minor (ARM repeats 6-8) binding sites (Fig. 7). Monopartite and bipartite cNLSs bind to the Impa binding sites in an extended conformation (Fig. 7). Structural analyses have demonstrated that monopartite cNLSs preferentially bind to the major binding site, reflected through lower crystallographic $B$-factors and the presence of more extensive electron density when compared to that observed at the minor binding site $[79,87,97]$. In addition, substitution of residues within the major binding site can abrogate nuclear accumulation of monopartite cNLS cargoes, whereas minor binding site mutations have minimal effects [106]. By contrast, mutation of residues at either minor or major binding sites can severely disrupt the interaction with bipartite cNLSs [106], which interact simultaneously with the two binding regions on the Impa surface.
The major binding site of Impa is composed of four principal binding cavities that bind the side chains of cNLS residues P2-P5 (Fig. 7). Structures of numerous cNLSs bound to Impa have been determined (Supplementary Table 2). For all characterized monopartite and bipartite sequences, the most crucial structural determinant is a Lys residue located at position P2. Its side chain forms a salt bridge with a highly conserved Impa Asp side chain (Fig. 7). Consistently, substitution of this conserved Asp residue results in an 300- to 400-fold decrease for monopartite and bipartite cNLS binding in ylmpa [106]. Although an Arg side chain can bind at the P2 position (PDB entry 4HTV; Supplementary Table 2), mutational studies on the SV40-TAg cNLS have demonstrated that a Lys is energetically favored at this position $[107,108]$. 
Although preference for long basic side chains is observed at the other major binding site positions (P3, P4 and P5), cNLSs can have a range of different amino acids in these positions (Supplementary Table $2)$. The calculated free-energy contributions of the SV40-TAg basic side chains at the P3-P5 positions are between 1/4 and 2/3 of that observed for the P2 Lys residue, with the $\mathrm{P} 4$ position contributing the least free energy to the interaction with Impa [107]. This suggests that non-basic side chains are not strictly necessary at the P3, P4 and P5 positions, provided that the overall affinity of the cNLS cluster is sufficient to constitute a functional cNLS motif. This is achieved by maximizing interactions at the other major binding site pockets, the regions directly flanking the major binding site or binding at the minor binding site in the case of bipartite cNLSs.

The two key amino acids in the $\mathrm{N}$-terminal region of bipartite cNLS (positions $\mathrm{P} 1^{\prime}-\mathrm{P} 2^{\prime}$ ) bind in a conserved manner to the minor NLS binding site, but adjacent auxiliary cavities can be used differentially in a cNLS-specific manner (Supplementary Table 2). In bipartite cNLSs, a "KR" motif is observed predominantly at these positions (Supplementary Table 2), with the P2' Arg side chain forming a salt bridge with a conserved Impa Glu residue. The total energetic contribution of the $\mathrm{P}^{\prime}{ }^{\prime}$ and $\mathrm{P} 2$ ' pockets has been calculated to be $3.2 \mathrm{kcal} / \mathrm{mol}$, comparable to that observed for a basic residue at the P3 or P5 position [107]. Although this interaction is modest, the addition of a KR motif $\mathrm{N}$-terminal to a non-functional SV40-TAg variant, whereby the critical P2 Lys residue was replaced with a Thr, was sufficient to direct nuclear accumulation of the protein [109]. Thus, compared to monopartite motifs, the sequence requirements at the major binding site are not as strict in bipartite cNLSs due to the additional interactions at the minor binding site, as well as cNLS-specific linker region interactions.

Structural studies showed that a minimum of 10 residues between the $\mathrm{P}^{\prime}$ ' and $\mathrm{P} 2$ positions is required to allow functional $\mathrm{CNLSs}$ to interact simultaneously with both the major and minor binding sites on the Impa surface [56]. Furthermore, early localization assays demonstrated that the linker region could tolerate non-conservative substitutions, as well as insertions [67], and these observations are consistent with the minimal interactions observed between bipartite cNLS linker regions and the Impa surface in crystal structures. Consistently, bipartite cNLS linker region residues are not well ordered and have higher crystallographic $B$-factors than residues at the major and minor binding sites. In some cNLSs, electron density is absent for most linker-region residues (Supplementary Table 2, residues in italics indicate residues not visible in crystallographic models), suggesting that bipartite linker regions longer than 12 residues likely bulge away from the Impa surface but are functional, provided that sufficient contacts at the major and minor binding regions are maintained. Notably, peptide library studies have demonstrated a preference for acidic residues in bipartite linker region sequences [110], and structural studies have shown that negatively charged side chains can form electrostatic interactions with the basic surface of Impa ARM repeats 4-6 [90].

Taken together, structural and biochemical data have revealed the molecular determinants of cNLS binding to the Impa adaptor. These studies have therefore enabled the elucidation of consensus sequences of both types of cNLSs. The monopartite cNLS motif is defined as $K(K / R) X(K / R)$, whereas the bipartite cNLS consensus sequences correspond to $\mathrm{KRX}_{10-12} \mathrm{KRRK}, \mathrm{KRX}_{10-12} \mathrm{~K}(\mathrm{KR})(\mathrm{KR})$ and $\mathrm{KR} \overline{\mathrm{X}_{10-12}} \mathrm{~K}(\mathrm{~K} / \mathrm{R}) \mathrm{X}(\mathrm{K} / \mathrm{R})$ (where $\mathrm{X}$ corresponds to any residue, Lys residues in boldface indicate the critical P2 lysine and minor site-binding KR motifs are underlined) $[65,90]$.

\section{Atypical Impa-dependent NLSs}

Several nuclear targeting signals are dissimilar to cNLSs described above but are nevertheless recognized by Impa. An analysis of binding of a random peptide library to Impa variants revealed six classes of NLSs, including two types of non-cNLSs, which were annotated "plant-specific" (consensus sequence LGKR[K/R][W/F/Y]) and "minor sitespecific" (consensus sequences $\mathrm{KRX}[\mathrm{W} / \mathrm{F} / \mathrm{Y}] \mathrm{XXAF}$ and $[R / P] X X K R[K / R][\wedge D E])$ NLSs [111]. Both of these types of NLSs feature a short basic cluster flanked C-terminally by hydrophobic residues.

Unique features of the binding of atypical NLSs to Impa have been identified by structural and biochemical studies. Crystal structures of mouse Impa in complex with poorly basic NLSs (G ${ }^{257}$ KISKHWTGI ${ }^{266}$; $\mathrm{G}^{273}$ SIIRKWN ${ }^{280}$ ) from the human phospholipid scramblase isoform hPLSCR1/4 show binding to the major and minor NLS binding sites, respectively $[95,99]$. The exclusive binding to the minor NLS binding site is also observed in naturally occurring NLSs \{e.g., the mouse RNA helicase II (Gua) NLS $\left(K^{842}\right.$ RSFSKAF $\left.\left.{ }^{849}\right)[101]\right\}$, whereas as the mitotic regulator protein, TPX2 NLS $\left(\mathrm{K}^{284} \mathrm{RKH}^{287}\right)$ binds predominantly to the minor site but could be considered an atypical bipartite NLS (with $\mathrm{K}^{327} \mathrm{MIK}^{330}$ binding to the major site) [91]. Unlike other NLSs that bind in an extended conformation, structural analysis of the Gua NLS and four other "minor site-specific" NLSs in complex with mouse Impa revealed that the C-terminal residues of these NLSs form an $\alpha$-helical turn [101]. This distinct structure of the NLS is stabilized by internal $\mathrm{H}$-bond and cation- $\mathrm{T}$ interactions between the aromatic residues from the NLSs and the positively charged residues from Impa. Such a conformation is prevented sterically at the Impa major binding site, explaining the minor site preference of 
these motifs [101]. Although contacts between "minor site-specific" NLSs are observed at the major binding site (Supplementary Table 2), the NLS peptides at the minor binding region have more extensive interactions and lower crystallographic $B$-factors.

Synthetic peptides corresponding to "plant-specific" NLSs show preferential binding to the minor NLS binding site of rice Impa, although the structural determinants of their binding mode are different from the ones observed for other "minor site-specific" NLSs [100]. Although putative naturally occurring "plantspecific" NLSs can be found using sequence analyses [100], they have not yet been characterized experimentally. Additional atypical NLSs have been identified that have not been characterized structurally, for example, in Borna disease virus $\mathrm{P} 10$ protein $\left(R^{6}{ }^{6}\right.$ TLLELVRRLNGN $\left.{ }^{19}\right)$ [112]. Bioinformatic analyses of the distribution of different classes of NLSs in diverse eukaryotes indicate that the atypical NLSs are much less prevalent than the monopartite and bipartite cNLSs [66].

\section{Structures of Impa in complex with native proteins}

Recognition of NLSs by Impa requires that these linear sequence motifs adopt an extended conformation. Consistently, cNLSs are located in disordered (and thus flexible) regions of native proteins and thus structural characterization of the Impa-cNLS interaction has predominantly involved the use of peptide sequences that correspond to NLS segments. Structural analyses of Impa in complex with native cNLScontaining proteins or domains have only been described for the influenza virus PB2 C-terminal fragment (residues 628-759) [83,84], the human cap-binding protein CBP80 (in complex with CBC20) [81] and the Ebola virus VP24 protein [113]. The PB2 C-terminal fragment has been crystallized in complex with four different human Impa isoforms (Impa5 [83] and Impa1, Impa3 and Impa7 [84]). In all these structures, a PB2 globular domain Lys residue located outside the canonical bipartite cNLS sequence interacts in trans with residues that comprise the Impa $\mathrm{P}^{\prime}$ pocket. In the absence of the globular domain, the PB2 bipartite cNLS is able to interact with the $\mathrm{P}^{\prime}$ '-binding pocket, causing a register shift in the cNLS minor binding site cavities (Supplementary Table 2) [84]. Differences in binding registers have also been described for structures of the SV40-TAg cNLS peptide (Supplementary Table 2), suggesting that these linear motifs can differentially bind to the Impa surface to maximize favorable interactions.

In contrast to the NLSs described above, the Ebola virus VP24 protein interacts with Impa through a distinctly different mechanism. The crystal structure of truncated human Impa (ARM repeats 7-10) in complex with VP24 shows that the virus protein primarily contacts the extreme C-terminus of the adaptor through three interspersed clusters on the surface of the folded VP24 structure [113]. Moreover, VP24 interacts with the opposite surface of Impa compared to NLS peptide sequences, with the $\mathrm{H} 2$ helices of ARM repeats 9 and 10 defining the VP24 interface [113]. Although the binding surfaces of NLS-containing cargo and VP24 do not overlap, a 2 -fold difference in binding is observed between the nucleoplasmin cNLS and Impa in the presence of VP24. This suggests that minor binding site interactions of cNLS sequences are allosterically affected by VP24 interaction with the outer Impa H2 helices [113].

Although bioinformatics analyses have suggested that a moderate proportion of ylmpa-binding proteins lack a detectable linear NLS [64], nuclear localization may also occur through "piggy-back" mechanisms, whereby translocation of a non-NLS-containing protein by Impa is mediated via interaction with an NLS-containing binding partner. The prevalence of proteins that mediate Impa binding through noncanonical means such as the VP24 protein is not known and cannot be identified by current cNLS prediction algorithms.

\section{Auto-inhibition by the Impa IBB domain}

The N-terminal IBB domain of Impa contains a cNLS-like sequence. Similar to cNLS motifs, the Impa IBB domain is rich in basic amino acids and interacts with the NLS binding pockets in the absence of cargo. In the mouse Impa structure, the IBB domain residues $\mathrm{K}^{49} \mathrm{RRN}^{52}$ are bound to the major NLS binding site (and correspond to cNLS positions P2-P5) [12]. This is consistent with lower affinity of cNLS binding to full-length Impa, compared to truncated Impa proteins that lack the IBB domain [114]. Likewise, increased affinity for cNLSs was observed when the corresponding $\mathrm{K}^{54} \mathrm{RR}^{56}$ motif in the IBB domain of yeast Impa was substituted with alanine residues [115]. This suggests that the IBB domain inhibits CNLS binding. In rice Impa, the $\mathrm{K}^{47} \mathrm{KRR}^{50}$ motif in the IBB domain was found to form analogous interactions with the major NLS binding site [100]. Distinct from the mouse structure, however, the $\mathrm{G}^{25} \mathrm{RRRR}^{29}$ motif in the rice Impa IBB domain additionally interacts with the minor NLS binding site. Similar to rice Impa, minor and major binding site interactions are observed between the IBB domain of the yeast protein and the solenoid domain when in complex with Cse1p and RanGTP [116]. The auto-inhibitory mechanism in plant and yeast Impa proteins may therefore differ from the mammalian proteins.

The IBB domain of Impa also mediates binding to $\operatorname{Imp} \beta 1$ and thus the interaction with $\operatorname{Imp} \beta 1$ promotes cNLS binding to Impa. The IBB domain-mediated auto-inhibitory mechanism presumably reduces futile import of empty adaptors and hinders cNLS binding 
when Imp $\beta 1$ is not present for nuclear translocation. Most structural studies of Impa binding to NLSs have therefore employed a truncated protein lacking the IBB domain.

\section{IBB domain-like NLSs}

A recent report describing the structures of the Heh1 and Heh2 inner membrane protein NLSs in complex with yeast Impa has revealed a bipartite mode of binding distinct from that observed in cNLSs [117]. Structural and biochemical analyses of these NLSs suggest similarities to the IBB domain interaction with the Impa adaptor: (1) the conformation of Heh2 NLS is similar to that observed for the IBB domain of full-length ylmpa in the auto-inhibited state (when in complex with RanGTP and Cse1p [116]); (2) mutational analyses have identified that the key structural determinant for these NLS motifs is the P2' pocket; this is in contrast to bipartite cNLS motifs, where nuclear accumulation is only modestly abrogated by disruption of P2' interaction [70,106]; and (3) pulldown assays demonstrate that the Heh1 and Heh2 NLS can efficiently relieve IBB domain auto-inhibition of Impa in the absence of $\operatorname{Imp} \beta 1$, unlike typical bipartite cNLS sequences [117]. However, in contrast to the Impa IBB domain, direct binding to Imp $\beta 1$ was not detected for the Heh1 and Heh2 NLSs [117]. Similar to the atypical monopartite NLSs, Heh1 and Heh2 mediate extensive contacts at the Impa minor binding site, with residues in this region having lower average $B$-factors compared to residues bound at the major binding pockets.

\section{Impa variants}

In some organisms, several Impa variants exist as a result of duplication events. The metazoan paralogues can be divided into three clades ( $\alpha 1, \alpha 2$ and $\alpha 3$ ); Impa proteins from Viridiplantae and Fungi belong to the $\alpha 1$-like clade [118-120]. The members of different subfamilies share $\sim 50 \%$ sequence identity, whereas within a subfamily, the identities are $>80 \%[118]$.

The structure and recognition mechanism are highly conserved among Impa proteins from different species [90,105] (Supplementary Fig. 1). However, Impa variants can display preferences for specific NLSs, which may be important for development and tissue-specific roles [118]. In D. melanogaster, which encodes three Impa proteins ( $\alpha 1, \alpha 2$ and $\alpha 3$ ), oogenesis depends on Impa2, and neither Impa1 nor Impa3 can substitute [121]. The mouse genome codes for six variants and Impa7 plays an essential role during the early stages of embryo development [122]. Some of the seven Impa variants in humans also display preferential interactions with specific cargoes, for example, Impa3 with RCC1 [123] and Impa5 for STAT proteins $[124,125]$.
The overall structure and the key NLS binding features of the different Impa variants are conserved in the crystal structures determined to date (Supplementary Table 3 and Fig. 7). Therefore, the reasons for specific NLS binding preferences by certain variants are not entirely clear but may relate to amino acid differences in the vicinity of NLS binding sites affecting NLS binding and auto-inhibition.

Structural analyses showed that plant-specific NLSs bind specifically to the minor NLS binding site of rice Impa but preferentially to the major site of mouse Impa [100], which has been attributed to specific amino acid differences in the C-terminal region of Impa. In particular, a Thr-to-Ser mutation prevents the binding of a plant-specific NLS peptide to the minor site of mouse Impa through steric hindrance. Indeed, it has been shown that SV40TAg NLS binding mode in the minor NLS site is not the same among Impa structures [105] (Supplementary Table 2). The structures of mouse and yeast Impa in complex with the SV40-TAg NLS peptide show "KK" residues at $\mathrm{P} 1^{\prime}-\mathrm{P} 2$ ' positions, whereas rice and N. crassa Impa structures show "KR" residues at these positions. The minor binding site may play a more important role in the a1-like Impa family, which includes the rice and $N$. crassa proteins.

The human is the only organism for which the structures of different Impa variants are available [83-85,113,126] (Supplementary Table 3). The overall structures and the NLS binding sites are conserved, consistent with the equivalent affinity in vivo of the influenza A PB2 NLS fragment for different Impa variants [84]. Differences identified between variants include a higher flexibility and reduced affinity between bipartite NLSs and Impa3, as well as different levels of auto-inhibition [84].

\section{Cargo release and recycling of Impa}

A consequence of the auto-inhibitory function of the Impa IBB domain is the facilitation of cargo release in the nucleus; once the trimeric complex has traversed the nuclear pore, dissociation of Imp $\beta 1$ from Impa in the nucleus allows the IBB domain to compete for the NLS binding site. In addition to this mechanism, some reports have implicated Nup50 (Nup2p in yeast) in Impa-cargo disassembly $[86,102,116]$. Solution-binding assays suggest that the addition of Nup2p can accelerate displacement of NLS cargo from the yeast protein [127]. Consistently, structures of Impa in complex with Nup50 peptide segments reveal interactions between the Nup side chains and the Impa minor binding site (Fig. 7E) [86,102,126]. In addition, contacts are observed outside the NLS binding region between the Nup and the Impa C-terminal region.

Impa is recycled back to the cytoplasm by its export factor CAS (Cse1 in yeast), which binds preferentially 
to cargo-free Impa [128]. Nuclear export of Impa further depends on RanGTP [129,130], and the formation of the trimeric CAS:Impa:RanGTP complex has been shown to be highly cooperative [128]. Binding of CAS-RanGTP to Impa displaces Nup50 through steric hindrance. Like other members of the $\beta$-Kap family, CAS has a superhelical HEAT repeat architecture and wraps around RanGTP and the Impa C-terminal region [116]. Extensive interactions are observed between the outer surfaces of Impa ARM repeats 8-10, in a region that overlaps with the VP24-binding site. Together, these mechanisms ensure that cargo is efficiently displaced from Impa and that recycling of the adaptor to the cytoplasm occurs only after cargo disassembly.

\section{Snurportin-Mediated Nuclear Import}

The nuclear import of assembled spliceosomal subunits, the uridine-rich small ribonucleoprotein particle UsnRNPs, employs a variation of the classical nuclear import pathway that utilizes a distinct adaptor protein termed snurportin-1. This protein, first identified via UV cross-linking to an $\mathrm{m}_{3} \mathrm{G}$-caped oligonucleotide, binds $\operatorname{Imp} \beta 1$ with an IBB domain similar to that found in Impa but lacks the canonical ARM repeat region [131], instead adopting a double $\beta$-sheet fold to form the $m_{3} G$-cap binding pocket [132]. Snurportin-1 binds both the hyper-methylated cap and the first nucleotide of the RNA in a stacking conformation, with the specificity determined by a highly solvent-exposed tryptophan [132].

\section{Symportin-1-mediated nuclear import}

Recent reports have described a new adaptor protein termed symportin-1 (Syo1 for synchronized import) that links nuclear cargo to Trn1 for nuclear translocation. Syo1 was identified through tandem affinity purification analysis of the yeast ribosomal protein Rpl5. Biochemical assays demonstrated direct binding of Syo1 to Rpl5 and the related protein Rpl11, as well as stable trimeric Syo1:Rpl5:Rpl11 complexes [133]. As Rpl5 and Rpl11 form a functional cluster within the ribosome, simultaneous binding to Syo1 suggested concomitant import of the ribosomal subunits, unlike other import pathways described to date that mediate binding of individual cargoes.

Syo1 is recognized by Trn1 through an N-terminal PY-NLS motif. The structure of Chaetomium thermophilum Syo1 revealed an unusual combination of four N-terminal ARM repeats fused to six C-terminal HEAT repeats in its globular cargo-binding domain

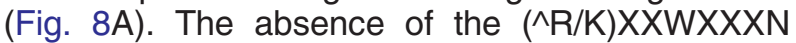
motif that forms the binding pockets on the Impa surface suggests that cNLS sequences are not able to bind to Syo1. However, structural characterization of the Syo1-Rpl5 peptide complex reveals that the inner concave surface of the Syo1 HEAT repeats mediates binding to the Rpl5 N-terminal region in a manner highly similar to the Impa-NLS interaction [133]. The Rpl5 peptide binds to Syo1 in an extended conformation with a short helical segment [133]. Conserved basic and aromatic residues throughout the Rpl5 linear motif mediate Syo1 binding. By contrast, the structure of the trimeric complex revealed that Rpl11 binding is mediated by the outer surface of
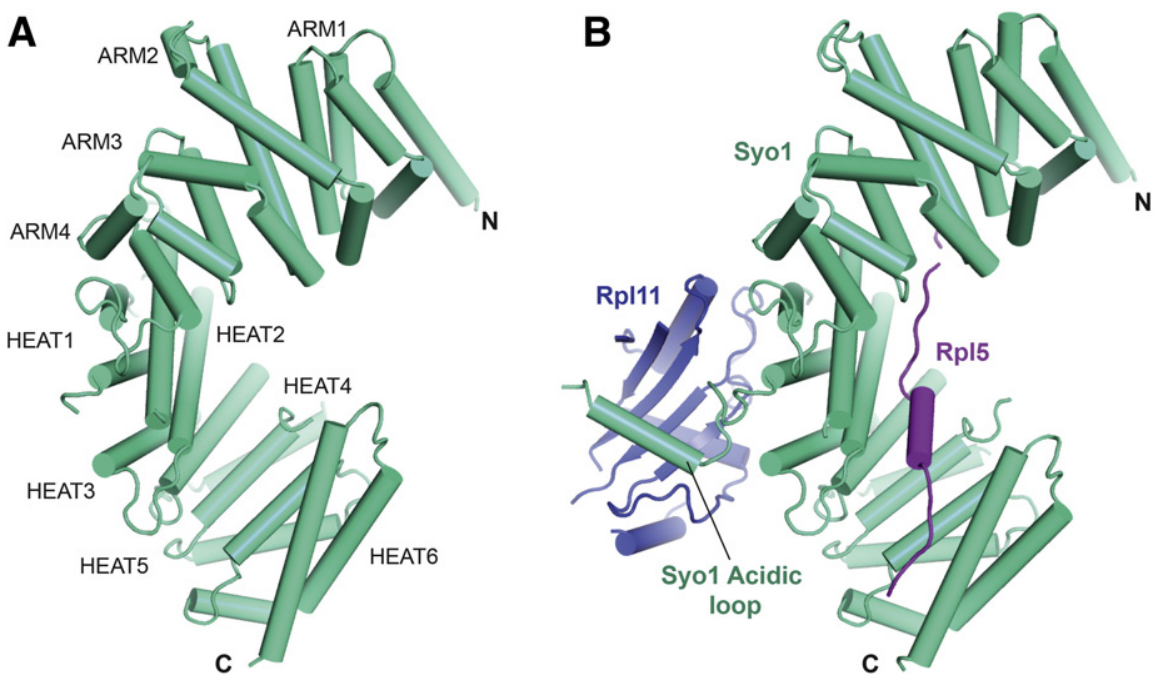

Fig. 8. The structure of symportin-1. (A) Structure of the $C$. thermophilum Syo1 adaptor (PDB entry 4GMO). The protein has an extended superhelical conformation composed of a unique chimera of four ARM (residues 65-260) and six HEAT (residues 274-675) repeats. (B) Structure of the Syo1:Rpl5:Rpl11 complex (PDB entry 5AFF). The Rpl5 peptide binds along the inner solenoid surface, while Rpl11 interacts with the outer surface of the Syo1 superhelix and a helical region from the HEAT repeat 1 acidic loop. 
the Syo1 superhelix, with additional contacts from a helical region in the large acidic loop of HEAT repeat 1 (Fig. 8B) [134].

\section{Other Nuclear Import Pathways}

Although most nuclear proteins depend on $\beta$-Kaps to reach their subcellular destination, some alternative pathways exist. These include the pathway operating during heat-shock stress that involves the carrier Hikeshi and the RanGDP import pathway that involves the nuclear transport factor NTF2 (see below). The actin-capping protein CapG also uses the interaction with NTF2 and Ran to enter the nucleus [135]. The RaDAR (RanGDP/ankyrin repeat) pathway has recently been characterized as an importinindependent nuclear import pathway for a number of ankyrin repeat proteins, with the signal identified as a hydrophobic residue at a specific position of two consecutive repeats [136]. The calcium-binding protein calmodulin can function as an import factor independent of $\beta$-Kaps, GTP and Ran, for a range of cargoes, particularly transcription factors [137-139]. Some proteins enter the nucleus independent of carrier molecules, for example, by direct binding to Nups, diffusion through the NPC and interaction with nuclear components (e.g., the ARM repeat protein $\beta$-catenin [140]). Lectins have been described as import factors for glycosylated proteins, and viruses disrupt the nuclear envelope during infection. Transport factors remaining to be characterized are involved in light-dependent nucleocytoplasmic trafficking in plants [141]. Some proteins can "piggy-back" through interactions with proteins with NLSs [142-145]. Many small proteins (e.g., histones) are imported by active transport mechanisms, although they could freely diffuse into the nucleus [146-149]. Proteins do not always use a single nuclear import pathway, which may be important under circumstances when conventional pathways are inhibited [150].

\section{Hikeshi-mediated nuclear import of Hsp70 proteins}

Nuclear import of heat-shock proteins from the Hsp70 family has been shown to be mediated by the nuclear transport factor Hikeshi [151] (see the review by Imamoto in this issue). The crystal structure of Hikeshi reveals a dimeric two-domain protein, with the $\mathrm{N}$-terminal domains responsible for the interaction with Nups [152]. The asymmetric nature of the dimer has been suggested to be important for the recognition of the ATP-bound form of Hsp70.

\section{NTF2-mediated nuclear import of RanGDP}

The conformational changes generated by nuclear RanGTP binding to $\beta$-Kaps lead to the release of their macromolecular cargo and adaptors (such as
Impa), but the karyopherins can only participate in another import cycle after Ran is released, following stimulation of its GTPase activity in the cytoplasm by RanGAP. The RanGDP generated in this way is then returned to the nucleus for recharging with GTP by the chromatin-bound RanGEF. Although Ran is a $25-k D a$ protein, its rate of nuclear import using simple diffusion appears to be insufficiently rapid to maintain adequate levels of karyopherin-based nuclear transport and is augmented by NTF2 [153,154] (reviewed in Ref. [155]).

The structure of NTF2 features an extensive $\beta$-sheet flanked by three helices, yielding a coneshaped molecule that dimerizes in solution (Fig. 9). The $\beta$-sheets of the protomers form an extensive interface in the NTF2 dimer, in which a considerable number of hydrophobic residues are buried [156,157]. The arrangement of the helices that flank the $\beta$-sheet generates an extensive cavity that is lined by
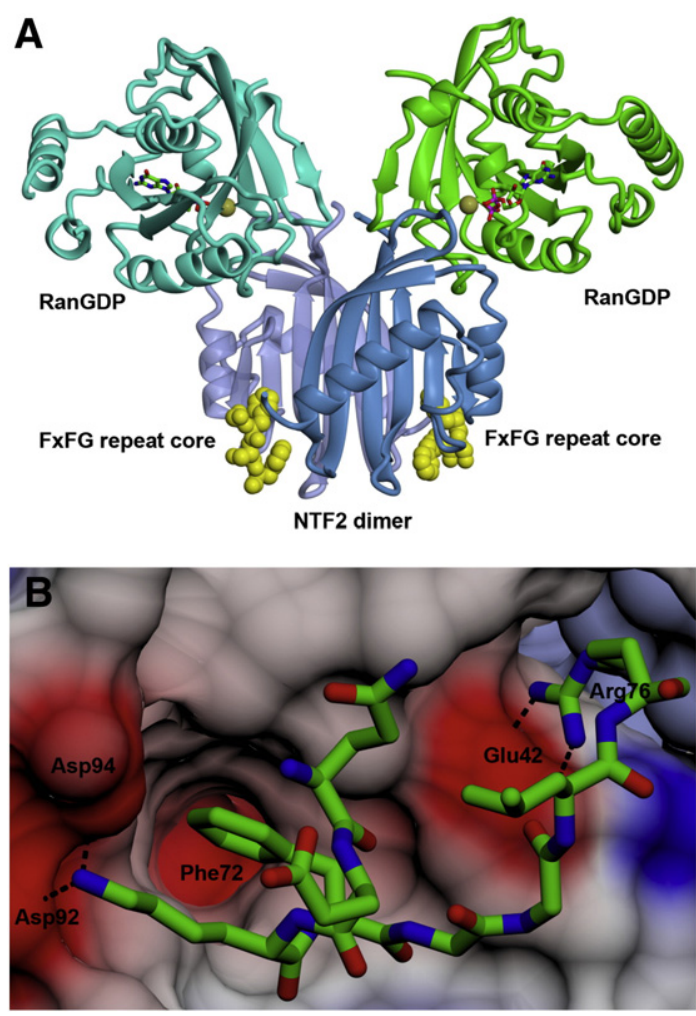

Fig. 9. NTF2 dimer (blue) bound to two chains of RanGDP (green) and two FxFG Nup motif cores (yellow) (based on PDB entries 5BXQ and 1GYB). (A) The two chains of the NTF2 dimer interact through an extensive $\beta$-sheet, whereas the remainder of the molecule generates a cavity into which Phe ${ }^{72}$ of the RanGDP switch-II loop binds. The FxFG motif cores bind in a hydrophobic cavity generated between the two NTF2 chains. (B) Binding of the RanGDP switch-II loop to NTF2. Ran Phe ${ }^{72}$ inserts into the hydrophobic cavity and is supplemented by salt bridges formed between Lys ${ }^{71}$ and Arg $^{76}$ of Ran and Asp ${ }^{92 / 94}$ and Glu $^{42}$ of NTF2, respectively. 
hydrophobic resides and that forms the principal interaction interface with RanGDP [158]. NTF2 recognizes the GDP-bound state of Ran through binding to the switch-II loop (Fig. 9). In the RanGDP conformation, $\mathrm{F}^{72}$ in the switch-ll loop inserts into the NTF2 cavity and this essentially hydrophobic interaction is complemented by salt bridges formed between $\mathrm{K}^{71}$ and $\mathrm{R}^{76}$ of Ran and $\mathrm{D}^{92} / \mathrm{D}^{94}$ and $\mathrm{E}^{42}$ of NTF2, respectively [158]. To mediate movement though the nuclear pore, NTF2 also binds to FxFG motifs present in many Nups, with the Phe residues of these motifs becoming buried in a hydrophobic cavity formed between the two chains in the dimer (Fig. 9) at a position opposite from that to which RanGDP binds $[40,159]$. The affinity of NTF2 for RanGDP is of the order of $100 \mathrm{nM}$ [157], which ensures that the dissociation rate of the NTF2:RanGDP complex is sufficiently slow for it to remain intact during nuclear transport, whereas the affinity of NTF2 for the Nup FxFG repeats is weaker $(\sim 5 \mu \mathrm{M})$, consistent with its forming much more rapidly dissociating complexes that enable the NTF2:RanGDP complex to move through the nuclear pore transport channel rapidly, using transient binding to Nups [159].

\section{Regulation of Nuclear Import Pathways}

One of the key features of limiting movement in and out of the nuclear compartment is the opportunity to regulate these transport processes. Regulation is essential for fine-tuning transport activities according to the actual cellular needs. Nucleocytoplasmic trafficking is regulated on several levels (see Refs. [160-162] for reviews), with new mechanisms continuing to be discovered (Fig. 10). The nuclear accumulation rate of cargoes is directly related to their binding affinities for their import receptors $[163,164]$ and thus transport processes can be regulated by modulating these binding affinities either by direct changes to the NLS or by physically blocking importin:cargo interactions through intermolecular or intramolecular NLS masking. Post-translational modification-induced changes are perhaps the best-described means of modulating transport processes. Although phosphorylation plays a central role, there is also a growing number of examples based on methylation or acetylation [165-169]. Post-translational modifications link nucleocytoplasmic transport to a variety of signaling pathways including the cell cycle, gene transcription, RNA metabolism, immune responses, apoptosis and the DNA damage response. Several of the case studies examined in the literature involve proteins that constantly shuttle in and out of the nucleus and the balance between nuclear import and export establishes the specific localization pattern. However, many of the mechanistic studies fail to investigate precisely how a post-translational modification perturbs the dynamics. For instance, if the post-translational modifications in the nucleus lead to increased cytoplasmic accumulation, this could result from changes in nuclear export (whether it is enhanced) or nuclear import (whether it is inhibited) or both.

\section{Regulation by phosphorylation}

\section{Modulation of importin:cargo binding affinity}

There are several examples in the literature of the introduction of a negative charge by phosphorylation inhibiting NLS binding $[160,161,170-172]$. Wellestablished examples include the phosphorylation of the yeast transcription factor Pho4, which disrupts the interaction with its dedicated carrier Pse1, an $\beta$-Kap family member [173,174], and the inhibitory phosphorylation by Cdk1 (cyclin-dependent kinase 1), which introduces negative charges that interfere with importin binding in a number of proteins $[93,175,176]$. In the case of the human dUTPase, structural work suggests that phosphorylation in the vicinity of the NLS leads to altered intra-NLS contacts that prevent favorable interactions with Impa, resulting in the cytoplasmic accumulation of the phosphorylated form [93]. The NLS:importin dissociation constants fall into a rather wide range $[107,177]$, and the effect of phosphorylation will depend on whether it is capable of moving the affinity over the threshold, so it falls outside the functional NLS range [65,90,107,177]. A high-affinity cargo complex would require a more substantial alteration to make the NLS non-functional.

Phosphorylation can also enhance nuclear accumulation through increasing NLS:Impa affinity. A well-established example is protein kinase CK2mediated phosphorylation of the SV40-TAg NLS at position $S^{111 / 112}$, which enhances affinity for Impa 2-fold, leading to a considerable increase in the nuclear import rate [178]. The nuclear transport efficiency also increases for the Epstein-Barr virus nuclear antigen 1 if its NLS is phosphorylated at $S^{385}$, which increases its affinity for Impa5. However, phosphorylation in two other neighboring positions, $\mathrm{S}^{383}$ and $\mathrm{S}^{386}$, decreases the nuclear import rate $[179,180]$. The precise structural reasons behind the enhanced affinity due to phosphorylation remain unclear [89]. Negative charges in the linker region of bipartite cNLSs were shown to have a positive effect on Impa binding. Generation of peptide inhibitors against the classical nuclear transport pathway led to bipartite NLSs that have several Glu or Asp residues in their linker regions. These help in maximizing the possible interactions in the cargo:carrier complex [90,110]. Clearly, phosphorylation has an effect specific to the position of the phosphorylated residue relative to the positive cluster of the NLS [175]. 
Post-translational modification-regulated transport

P phosphorylation (AC) acetylation (10) methylation (16) ubiquitination (SU) sumoylation

(A) Affinity modulation

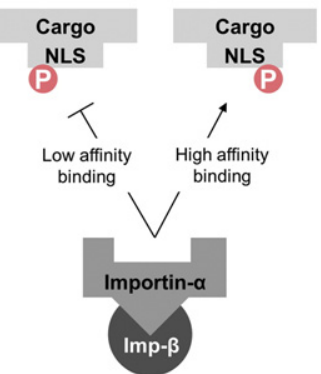

Other mechanisms affecting nucleo-cytoplasmic trafficking
(B) Intra-molecular masking

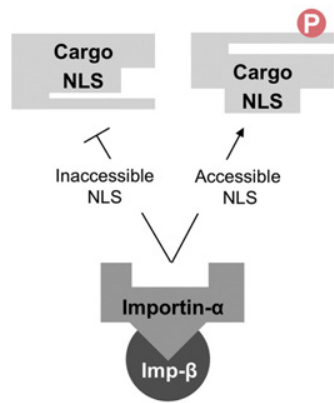

(C) Inter-molecular masking

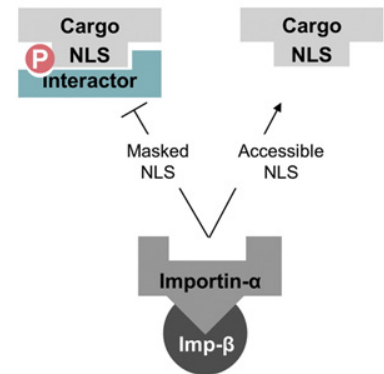

(D) Microtubular transport

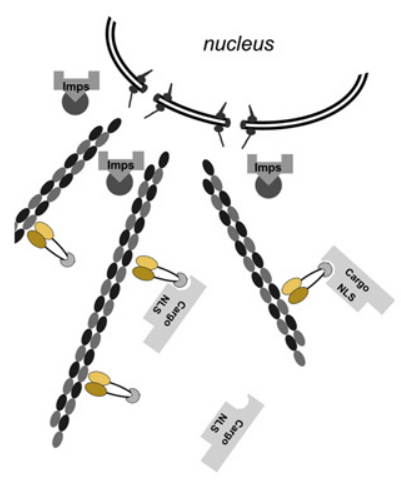

(E) Piggy-back mechanism

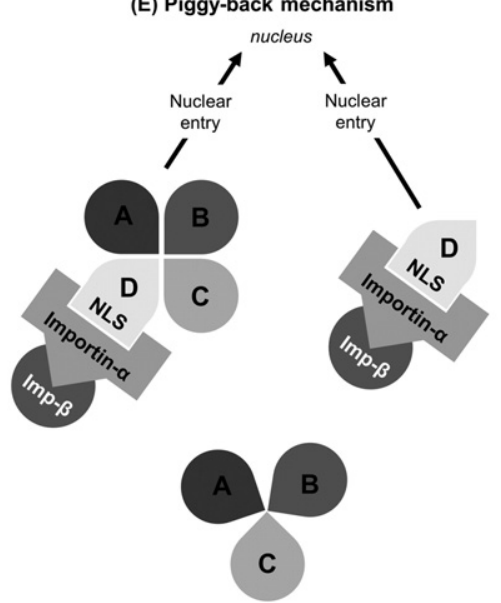

(F) NLS copy number variation

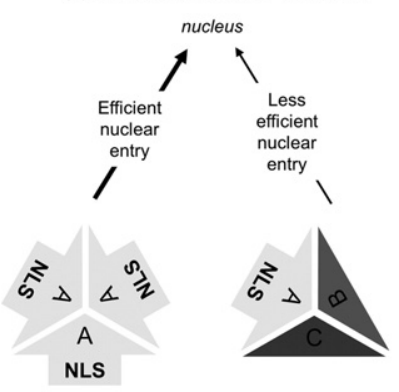

Fig. 10. Regulatory mechanisms in nucleocytoplasmic trafficking. The control of trafficking in and out of the nucleus at the protein level operates by several types of mechanisms. First, different chemical moieties can be attached to the cargo or transport factors, as shown in the first row. These post-translational modifications may alter the thermodynamics and kinetics of the interactions between the cargo and the karyopherin $(A)$ or may lead to masking of the interacting groups ( $B$ and $C)$. Other mechanisms involve the microtubular system, which can influence the concentration gradient of cargo proteins such that these may accumulate around the nuclear pores where importins are readily available (D). "Piggy-backing" is the indirect coupling between cargo and karyopherins (E). NLS copy number variation for oligomeric cargo proteins constitutes a fine-tuning control that provides advantage to homo-oligomers or hetero-oligomers with an increased number of NLS segments $(F)$.

\section{Intramolecular NLS masking}

Intramolecular NLS masking can also inhibit cargo:carrier complex formation, through induced structural changes in the cargo making the NLS inaccessible to Impa. In the case of the $X$. laevis b-Myb protein, the C-terminal domain simultaneously inhibits DNA binding and NLS function. During embryo development, b-Myb is subjected to several modifications, which result in the NLSs that facilitate nuclear accumulation of the protein becoming accessible [181]. STAT1 activation through the phosphorylation of a tyrosine residue $\left(\mathrm{Y}^{701}\right)$ is one of the central events in cytokine signaling and the regulation of immune responses. Phosphorylation induces a structural rearrangement that shifts STAT1 dimers from an antiparallel to a parallel conformation, exposing a non-classical NLS that is recognized by Impa5. Dimerization and phosphorylation are essential for efficient nuclear accumulation during STAT1 activation, even though the phospho-tyrosine residue is not a binding determinant for Impa5 [124,180,182-185].

\section{Intermolecular NLS masking and organelle-specific retention}

Intermolecular masking can occur if the binding of a heterologous protein prevents the interaction of the 
cargo and its carrier. One of the best-described examples is the NF-kB p50/p65 heterodimer, a transcription factor regulating immune and stress responses, apoptosis and differentiation. NF-KB is kept inactive by its inhibitor, IkBa, which impedes NF-KB from being recognized by the nuclear import machinery. Crystal structures show how the NLSs of both NF-kB p50 and p65 subunits are covered by the ankyrin repeat region of IKBa $[186,187]$. IkBa binds the NLS of NF-KB until phosphorylation licenses its ubiquitin-mediated degradation, which enables Impa3 and Impa4 to access the unmasked NF-KB NLSs [188-191].

$A$ recently described E3 ubiquitin ligase that binds phospho-NLSs, the BRCA1-binding protein BRAP2, was shown to reduce the nuclear accumulation of several viral and endogenous proteins, depending on their phosphorylation state. Although it does not completely sequester its targets in the cytoplasm, it fine-tunes their localization pattern [192,193].

DNA or RNA can also be responsible for intermolecular masking. For example, the DNA binding region and the Imp $\beta 1$-recognized NLS of the human sex-determining factor SRY overlap. DNA binding inhibits Imp $\beta 1$ binding and vice versa. This mechanism may also facilitate the release of the SRY:Imp $\beta 1$ complex once it enters the nucleus [194]. Interestingly, acetylation of SRY is necessary for proper Imp $\beta 1$ :SRY complex formation, showing the interplay among different modes of regulation [195]. The NLS of HDAC4, a class-Ila histone deacetylase, is masked by phosphorylation-induced 14-3-3 protein binding, making both NLSs inaccessible to nuclear import factors and causing cytoplasmic retention [196]. A similar mechanism seems to apply for HDAC5, HDAC7 and HDAC9, where phosphorylation near the NLSs, along with 14-3-3 binding, inhibits nuclear translocation [197].

\section{Regulation by methylation and acetylation}

Post-translational modifications of histones, including acetylation and methylation, are crucial in epigenetics. A growing number of examples show that acetylation and methylation of Lys or Arg residues, in addition to those in histones, regulates a variety of cellular functions [198-200]. Interestingly, Impa itself is targeted for acetylation within its IBB domain by $\mathrm{p} 300 / \mathrm{CBP}$, increasing its ability to bind Imp $\beta 1$ in vitro [201]. p300/CBP interacts with several components of the nuclear transport machinery, possibly fine-tuning their functions by affecting their intracellular distribution [202]. Methylation and acetylation of Lys and Arg residues can directly modulate NLS/NES function through altering the interaction with the transport machinery by modulating the residue charge. These modifications may also have indirect effects on localization through alteration of binding between interaction partners or by promoting conformational changes. Although the precise mechanisms are mostly unclear [203], several well-documented examples are summarized below.

\section{Modulation of importin:cargo binding affinity}

In c-Abl, lysine acetylation occurs in the NLS. The protein is involved in apoptosis when nuclear, whereas it responds to proliferative signals in the cytoplasm. It can be acetylated in the nucleus by P/CAF (an acetyltransferase) within one of its NLSs, leading to cytoplasmic accumulation. It is hypothesized that when the acetylated form is exported to the cytoplasm, it cannot re-enter the nucleus because its NLS is no longer recognized by the import machinery [204]. A similar mechanism was proposed for RECQL4 (a DNA helicase important for genomic integrity maintenance), which is acetylated by p300 [205], as well as for HMGB1 (a protein involved in transcriptional control) [206]. Acetylation within the NLS of the poly $(A)$ polymerase PAP leads to its cytoplasmic accumulation. Acetylation directly interferes with PAP binding to Impa/Imp $\beta 1$ [207]. In the case of another P/CAF substrate, E1A, a clear negative effect of NLS acetylation on Impa3 binding has been demonstrated [208]. Interestingly, for $\mathrm{P} / \mathrm{CAF}$ itself, intramolecular acetylation is required for its nuclear localization. However, a mutant incapable of auto-acetylation is strictly cytoplasmic despite the fact that the acetylated form of P/CAF shows decreased binding to both Impa1 and Imp $\beta 1$ in in vitro pull-down assays, compared to non-acetylated P/CAF. Acetylation possibly affects the P/CAF localization pattern through regulation of proteasomedependent degradation processes [209]. Besides acetylation, phosphorylation also influences the localization of P/CAF. Phosphorylation may promote the dissociation of P/CAF-PP1/PP2a cytoplasmic complexes and may allow the nuclear import of P/CAF [210]. Although the precise mechanism is not yet known, acetylation near the NLSs of Net1A (a RhoA GEF protein) alters the dynamics of its nucleocytoplasmic shuttling, probably by slowing its nuclear re-import rate, leading to cytoplasmic accumulation [211]. Acetylation can also directly enhance importin:cargo interactions. It has been shown that p300-mediated acetylation in the proximity of the NLS of SRY $\left(\mathrm{K}^{136}\right)$ is needed for nuclear localization, facilitating Imp $\beta 1$ binding [195].

\section{Intramolecular NLS masking}

Conformational changes induced by acetylation can alter nucleocytoplasmic transport processes in a fashion similar to phosphorylation. The transcription factor HNF-4 shuttles between the nuclear and cytoplasmic compartments and CBP-mediated acetylation is hypothesized to induce conformational 
changes that make the NES inaccessible to CRM1. Acetylation also enhances the ability of HNF-4 to bind DNA and CBP, both of which help its nuclear anchoring [212]. One of the key aspects of the many modes of regulation of p53 localization is its phosphorylation- and acetylation-dependent tetramerization, which influences the accessibility of its NES and NLSs (reviewed in Refs. [213] and [214]). Acetylation of lysine residues in the C-terminal region of p53 inhibits its oligomerization, resulting in an exposed NES and effective nuclear export [215]. By contrast, CBP-dependent acetylation of survivin at $\mathrm{K}^{129}$ enhances its oligomerization, making its NES sequence inaccessible to CRM1 and leading to nuclear accumulation of the protein $[216,217]$. The acetylation of $\mathrm{K}^{433}$ in the protein kinase PKM2 by p300 is speculated to influence its nuclear transport through stabilizing its dimeric state because the tetrameric state may bury its NLS [218] or by modulating a "piggy-back" nuclear entry mechanism of PKM2.

\section{Intermolecular NLS masking and organelle-specific retention}

The localization of Yap, one of the key components of the Hippo signaling pathway, is regulated through lysine methylation. Yap is monomethylated at $\mathrm{K}^{494}$ by Set7, which leads to its cytoplasmic retention though an unknown mechanism [219]. Hsp70, a protein with roles in folding and degradation, mainly localizes to the nucleus when dimethylated on $\mathrm{K}^{561}$, whereas the unmethylated form is predominantly cytoplasmic. A small fraction of the overall Hsp70 pool was reported to be dimethylated in cancer cells and this form is thought to interact specifically with Aurora kinase $B$, which might be responsible for the chromatin association of methylated Hsp70 [220]. RNA helicase A shuttles between the nuclear and cytoplasmic compartments, and PRMT1-mediated arginine methylation is hypothesized to inhibit its interaction with a putative cytoplasmic retention factor binding its NLS-containing C-terminal region [221]. CtBP2 is actively exported from the nucleus in a CRM1-dependent manner, but this is prevented by $\mathrm{K}^{10}$ acetylation-dependent nuclear sequestration, mediated by p300 [222]. Interestingly, the localization of its closely related protein, CtBP1, is regulated by sumoylation (causing enhanced nuclear entry or retention) [223], cytoplasmic retention [224] and phosphorylation [225]. Finally, acetylation of the retinoblastoma protein $\mathrm{Rb}$ by P/CAF was shown to be important for $\mathrm{Rb}$ to remain nuclear during keratinocyte differentiation. Because acetylation presumably happens in the nucleus, it does not have an effect on nuclear import, although the modification is within the NLS [226].

\section{Regulation by ubiquitination and sumoylation}

Other post-translational covalent modifications, including ubiquitination or sumoylation, can also regulate protein nuclear import. These modifications can directly target the Lys residues in the NLS. A well-documented example involves cytidylyltransferase, a protein involved in phosphatidylcholine biosynthesis. Monoubiquitination on $\mathrm{K}^{57}$ masks the NLS of cytidylyltransferase, resulting in cytoplasmic accumulation [227]. By a similar mechanism, ubiquitinated $\mathrm{K}^{319-321}$ in p53 blocks Impa3 binding, inhibiting nuclear entry [228]. Nuclear entry of PTEN, the regulator of phosphatidylinositol 3-kinase signaling, is essential for its tumor suppressor function and this translocation depends on NEDD4-1-mediated monoubiquitination of two lysine residues $\left(\mathrm{K}^{13}\right.$ and $\left.\mathrm{K}^{289}\right)$ [229-231]. Sumoylation of PAP within its NLS is essential for its nuclear import, providing regulation additional to acetylation [232]. Sumoylation can also enhance nuclear accumulation through masking NESs as in the case of Kfl5, a transcription factor regulating cell proliferation [233], or through supporting nuclear retention without altering nuclear import dynamics as in the case of SAE1 (SUMO activating enzyme 1) [234]. Nuclear translocation of the enzymes in the de novo thymidylate biosynthesis pathway is sumoylation dependent and takes place at the beginning of S-phase [235-237].

\section{Other factors regulating nucleocytoplasmic trafficking}

Among several other possible ways to regulate nuclear translocation, cytoplasmic anchoring and the contribution of the microtubular network are important for a number of proteins. The microtubular system and its associated molecular motors are essential for some viral proteins to reach the nucleus to overcome barriers to diffusion (reviewed in Refs. [238] and [239]), but several non-viral proteins also use them, presumably to enhance the rate and extent of their nuclear import. Nocodazole (a tubulin polymerization inhibitor) treatment of cells significantly reduces the nuclear accumulation of p53 [240], Rb [241] and PTHrP [242,243]. These results show that NLS-containing cargoes can be transported actively to sites close to the NPCs, enhancing nuclear entry rates by moving cargoes to intracellular regions where the $\operatorname{Imp} \beta 1$ concentrations are high $[244,245]$. This may enhance the response time of cells to extracellular or intracellular stimuli when rapid transport processes are needed for proper function (e.g., in cell signaling and DNA damage responses).

The nuclear localization of several steroid receptors, including the glucocorticoid and estrogen receptors, is regulated through cytoplasmic retention. Without their ligands, these receptors are sequestered in the cytoplasm by Hsp90 through 
their ligand-binding domains. Upon ligand binding, they release Hsp90 and are imported into the nucleus using their NLSs [246]. The androgen receptor is similarly bound to importin-7 (Imp7), which in the absence of ligand inhibits binding of Impa to the NLS and causes the receptor to remain in the cytoplasm. Androgen binding induces conformational changes in the receptor that result in the release of Imp7, exposing the NLS for binding to Impa and leading to translocation into the nucleus [247].

In addition to the affinity for their receptors, the NLS copy number also has an important effect on nuclear accumulation efficiency. Higher numbers of NLSs provide advantage in competing for importins in the cellular environment, making oligomerizationinduced NLS copy number variations another way of regulating nucleocytoplasmic transport processes $[248,249]$.

\section{Conclusions}

Although the first components of nuclear import pathways were only identified in 1993 [250,251], there is now a substantial molecular understanding of how many of the nuclear protein import pathways function. However, several key challenges remain, including determining the precise mechanism by which the cargo:carrier complex is able to overcome the barrier function generated by the Nups to facilitate translocation through the NPC, accurate prediction of nuclear targeting signals from protein sequences, defining the catalytic role of Nups on the cytoplasmic and nuclear faces of NPCs in transport complex assembly and disassembly, characterizing the molecular mechanisms or regulation of nucleocytoplasmic transport, determining the structures of complexes of karyopherins with full-length cargo proteins and targeting the components of nuclear transport machinery therapeutically. Examples of $\beta$-Kap family proteins involved in nuclear import with no structural information available currently include importin-7 and importin-8 $[252,253]$. Structural biology has played a key role in characterizing the molecular events in nuclear import pathways and will no doubt continue to do so in the future. Recent advances in cryo-electron microscopy [254] and X-ray free electron lasers [255], to name just two, should help characterize the structures of large and dynamic multi-protein complexes that form during nuclear import.

\section{Acknowledgements}

The research in the authors' laboratories has been supported by the National Health and Medical Research Council Program (1000512 and 565526),
Project (1003326) and Research Fellowship (1003325) and Australian Research Council Discovery Project (DP120100685) to B.K.; National Health and Medical Research Council Fellowship to A.G.S. (1090408); UK Medical Research Council Grant U105178939 to M.S.; Hungarian Scientific Research Fund OTKA (NK 84008 and K109486), Baross Program of the New Hungary Development Plan (3DSTRUCT, OMFB-00266/2010 REG-KM-091-2009-0050) and Hungarian Academy of Sciences (TTK IF-28/ 2012 and ICGEB CRP/HUN14-01) to B.G.V.; Young Researcher Fellowships from the Hungarian Academy of Sciences to G.R.; Conselho Nacional de Desenvolvimento Científico e Tecnológico, Fundação de Amparo à Pesquisa do Estado de São Paulo and Coordenação de Aperfeiçoameno de Pessoal de Nível Superior to M.R.M.F.; and Australian Research Council Future Fellowship to J.K.F. (100095). We acknowledge the use of the University of Queensland Remote Operation Crystallization and $X$-ray Diffraction Facility and the Australian Synchrotron.

\section{Appendix A. Supplementary data}

Supplementary data to this article can be found online at http://dx.doi.org/10.1016/j.jmb.2015.10.023.

Received 17 July 2015; Received in revised form 16 October 2015; Accepted 24 October 2015 Available online 30 October 2015

$$
\begin{array}{r}
\text { Keywords: } \\
\text { beta-karyopherin; } \\
\text { crystal structure; } \\
\text { importin-alpha; } \\
\text { nuclear localization signal (NLS); } \\
\text { nucleocytoplasmic transport }
\end{array}
$$

$\uparrow$ M.C., C.-W.C., G.R. and K.M.S. contributed equally to this work.

\section{Abbreviations used:}

IBB, importin- $\beta$ binding; Impa, importin- $\alpha$; Imp $\beta 1$, importin$\beta$; Imp13, importin-13; $\beta$-Kap, $\beta$-karyopherin; NLS, nuclear localization signal; Trn1, transportin-1; Trn3, transportin-

3; NPC, nuclear pore complex; Nup, nucleoporin; cNLS, classical nuclear localization sequence; NES, nuclear export signal.

\section{References}

[1] F. Alber, S. Dokudovskaya, L.M. Veenhoff, W. Zhang, J. Kipper, D. Devos, et al., The molecular architecture of the nuclear pore complex, Nature 450 (2007) 695-701. 
[2] J.D. Aitchison, R.W. Wozniak, Cell biology: Pore puzzle, Nature 450 (2007) 621-622.

[3] M.A. Andrade, P. Bork, HEAT repeats in the Huntington's disease protein, Nat. Genet. 11 (1995) 115-116.

[4] B. Kobe, A.V. Kajava, When protein folding is simplified to protein coiling: The continuum of solenoid protein structures, Trends Biochem. Sci. 25 (2000) 509-515.

[5] D. Gorlich, N. Pante, U. Kutay, U. Aebi, F.R. Bischoff, Identification of different roles for RanGDP and RanGTP in nuclear protein import, EMBO J. 15 (1996) 5584-5594.

[6] F.R. Bischoff, H. Ponstingl, Catalysis of guanine nucleotide exchange on Ran by the mitotic regulator RCC1, Nature 354 (1991) 80-82.

[7] F.R. Bischoff, C. Klebe, J. Kretschmer, A. Wittinghofer, H. Ponstingl, RanGAP1 induces GTPase activity of nuclear Ras-related Ran, Proc. Natl. Acad. Sci. U. S. A. 91 (1994) 2587-2591.

[8] J. Moroianu, G. Blobel, A. Radu, Nuclear protein import: Ran-GTP dissociates the karyopherin alpha-beta heterodimer by displacing alpha from an overlapping binding site on beta, Proc. Natl. Acad. Sci. U. S. A. 93 (1996) 7059-7062.

[9] M. Rexach, G. Blobel, Protein import into nuclei: Association and dissociation reactions involving transport substrate, transport factors, and nucleoporins, Cell 83 (1995) 683-692.

[10] N.C. Chi, E.J. Adam, G.D. Visser, S.A. Adam, RanBP1 stabilizes the interaction of Ran with p97 nuclear protein import, J. Cell Biol. 135 (1996) 559-569.

[11] D. Gorlich, F. Vogel, A.D. Mills, E. Hartmann, R.A. Laskey, Distinct functions for the two importin subunits in nuclear protein import, Nature 377 (1995) 246-248.

[12] B. Kobe, Autoinhibition by an internal nuclear localization signal revealed by the crystal structure of mammalian importin alpha, Nat. Struct. Biol. 6 (1999) 388-397.

[13] E. Conti, J. Kuriyan, Crystallographic analysis of the specific yet versatile recognition of distinct nuclear localization signals by karyopherin alpha, Structure 8 (2000) 329-338.

[14] U. Kutay, S. Guttinger, Leucine-rich nuclear-export signals: Born to be weak, Trends Cell Biol. 15 (2005) 121-124.

[15] A. Cook, F. Bono, M. Jinek, E. Conti, Structural biology of nucleocytoplasmic transport, Annu. Rev. Biochem. 76 (2007) 647-671.

[16] Y.M. Chook, K.E. Suel, Nuclear import by karyopherinbetas: Recognition and inhibition, Biochim. Biophys. Acta 2011 (1813) 1593-1606.

[17] Y. Quan, Z.L. Ji, X. Wang, A.M. Tartakoff, T. Tao, Evolutionary and transcriptional analysis of karyopherin beta superfamily proteins, Mol. Cell. Proteomics 7 (2008) 1254-1269.

[18] M.A. Andrade, C. Petosa, S.I. O'Donoghue, C.W. Muller, P. Bork, Comparison of ARM and HEAT protein repeats, J. Mol. Biol. 309 (2001) 1-18.

[19] B. Kobe, T. Gleichmann, J. Horne, I.G. Jennings, P.D. Scotney, T. Teh, Turn up the HEAT, Structure 7 (1999) R91-R97.

[20] H.S. Malik, T.H. Eickbush, D.S. Goldfarb, Evolutionary specialization of the nuclear targeting apparatus, Proc. Natl. Acad. Sci. U. S. A. 94 (1997) 13738-13742.

[21] J.K. Forwood, A. Lange, U. Zachariae, M. Marfori, C. Preast, H. Grubmuller, et al., Quantitative structural analysis of importin-beta flexibility: Paradigm for solenoid protein structures, Structure 18 (2010) 1171-1183.

[22] G. Cingolani, C. Petosa, K. Weis, C.W. Muller, Structure of importin-beta bound to the IBB domain of importin-alpha, Nature 399 (1999) 221-229.
[23] G. Cingolani, J. Bednenko, M.T. Gillespie, L. Gerace, Molecular basis for the recognition of a nonclassical nuclear localization signal by importin beta, Mol. Cell 10 (2002) 1345-1353.

[24] S.J. Lee, T. Sekimoto, E. Yamashita, E. Nagoshi, A. Nakagawa, N. Imamoto, et al., The structure of importinbeta bound to SREBP-2: Nuclear import of a transcription factor, Science 302 (2003) 1571-1575.

[25] S. Choi, E. Yamashita, N. Yasuhara, J. Song, S.Y. Son, Y.H. Won, et al., Structural basis for the selective nuclear import of the $\mathrm{C} 2 \mathrm{H} 2$ zinc-finger protein Snail by importin beta, Acta Crystallogr. D Biol. Crystallogr. 70 (2014) 1050-1060.

[26] K. Lott, G. Cingolani, The importin beta binding domain as a master regulator of nucleocytoplasmic transport, Biochim. Biophys. Acta 2011 (1813) 1578-1592.

[27] I.R. Vetter, A. Arndt, U. Kutay, D. Gorlich, A. Wittinghofer, Structural view of Ran-importin beta interaction at $2.3 \AA$ resolution, Cell 97 (1999) 635-646.

[28] S.J. Lee, Y. Matsuura, S.M. Liu, M. Stewart, Structural basis for nuclear import complex dissociation by RanGTP, Nature 435 (2005) 693-696.

[29] J.K. Forwood, T.G. Lonhienne, M. Marfori, G. Robin, W. Meng, G. Guncar, et al., Kap95p binding induces the switch loops of RanGDP to adopt the GTP-bound conformation: Implications for nuclear import complex assembly dynamics, J. Mol. Biol. 383 (2008) 772-782.

[30] I.R. Vetter, C. Nowak, T. Nishimoto, J. Kuhlmann, A. Wittinghofer, Structure of a Ran-binding domain complexed with Ran bound to a GTP analogue: Implications for nuclear transport, Nature 398 (1999) 39-46.

[31] F.R. Bischoff, D. Gorlich, RanBP1 is crucial for the release of RanGTP from importin beta-related nuclear transport factors, FEBS Lett. 419 (1997) 249-254.

[32] M. Saric, X. Zhao, C. Korner, C. Nowak, J. Kuhlmann, I.R. Vetter, Structural and biochemical characterization of the Importin-beta.Ran.GTP.RanBD1 complex, FEBS Lett. 581 (2007) 1369-1376.

[33] M.J. Seewald, C. Korner, A. Wittinghofer, I.R. Vetter, RanGAP mediates GTP hydrolysis without an arginine finger, Nature 415 (2002) 662-666.

[34] F.R. Bischoff, H. Krebber, E. Smirnova, W. Dong, H. Ponstingl, Co-activation of RanGTPase and inhibition of GTP dissociation by Ran-GTP binding protein RanBP1, EMBO J. 14 (1995) 705-715.

[35] M.J. Seewald, A. Kraemer, M. Farkasovsky, C. Korner, A. Wittinghofer, I.R. Vetter, Biochemical characterization of the Ran-RanBP1-RanGAP system: Are RanBP proteins and the acidic tail of RanGAP required for the RanRanGAP GTPase reaction? Mol. Cell. Biol. 23 (2003) 8124-8136.

[36] K. Scheffzek, M.R. Ahmadian, W. Kabsch, L. Wiesmuller, A. Lautwein, F. Schmitz, et al., The Ras-RasGAP complex: Structural basis for GTPase activation and its loss in oncogenic Ras mutants, Science 277 (1997) 333-338.

[37] K. Rittinger, P.A. Walker, J.F. Eccleston, K. Nurmahomed, D. Owen, E. Laue, et al., Crystal structure of a small G protein in complex with the GTPase-activating protein rhoGAP, Nature 388 (1997) 693-697.

[38] C.I. Villa Braslavsky, C. Nowak, D. Gorlich, A. Wittinghofer, J. Kuhlmann, Different structural and kinetic requirements for the interaction of Ran with the Ran-binding domains from RanBP2 and importin-beta, Biochemistry 39 (2000) $11629-11639$. 
[39] J. Kuhlmann, I. Macara, A. Wittinghofer, Dynamic and equilibrium studies on the interaction of Ran with its effector, RanBP1, Biochemistry 36 (1997) 12027-12035.

[40] R. Bayliss, T. Littlewood, L.A. Strawn, S.R. Wente, M. Stewart, GLFG and FxFG nucleoporins bind to overlapping sites on importin-beta, J. Biol. Chem. 277 (2002) 50597-50606.

[41] S.M. Liu, M. Stewart, Structural basis for the high-affinity binding of nucleoporin Nup1p to the Saccharomyces cerevisiae importin-beta homologue, Kap95p, J. Mol. Biol. 349 (2005) 515-525.

[42] J. Bednenko, G. Cingolani, L. Gerace, Importin beta contains a $\mathrm{COOH}$-terminal nucleoporin binding region important for nuclear transport, J. Cell Biol. 162 (2003) 391-401.

[43] T.A. Isgro, K. Schulten, Binding dynamics of isolated nucleoporin repeat regions to importin-beta, Structure 13 (2005) 1869-1879.

[44] B.J. Lee, A.E. Cansizoglu, K.E. Suel, T.H. Louis, Z. Zhang, Y.M. Chook, Rules for nuclear localization sequence recognition by karyopherin beta 2, Cell 126 (2006) 543-558.

[45] N. Bonifaci, J. Moroianu, A. Radu, G. Blobel, Karyopherin beta2 mediates nuclear import of a mRNA binding protein, Proc. Natl. Acad. Sci. U. S. A. 94 (1997) 5055-5060.

[46] V.W. Pollard, W.M. Michael, S. Nakielny, M.C. Siomi, F. Wang, G. Dreyfuss, A novel receptor-mediated nuclear protein import pathway, Cell 86 (1996) 985-994.

[47] R.A. Fridell, R. Truant, L. Thorne, R.E. Benson, B.R. Cullen, Nuclear import of hnRNP A1 is mediated by a novel cellular cofactor related to karyopherin-beta, J. Cell Sci. 110 (1997) $1325-1331$.

[48] A.E. Cansizoglu, B.J. Lee, Z.C. Zhang, B.M. Fontoura, Y.M. Chook, Structure-based design of a pathway-specific nuclear import inhibitor, Nat. Struct. Mol. Biol. 14 (2007) 452-454.

[49] T. Imasaki, T. Shimizu, H. Hashimoto, Y. Hidaka, S. Kose, N. Imamoto, et al., Structural basis for substrate recognition and dissociation by human transportin 1, Mol. Cell 28 (2007) $57-67$.

[50] Z.C. Zhang, Y.M. Chook, Structural and energetic basis of ALS-causing mutations in the atypical proline-tyrosine nuclear localization signal of the Fused in Sarcoma protein (FUS), Proc. Natl. Acad. Sci. U. S. A. 109 (2012) 12017-12021.

[51] M. Soniat, P. Sampathkumar, G. Collett, A.S. Gizzi, R.N. Banu, R.C. Bhosle, et al., Crystal structure of human karyopherin beta2 bound to the PY-NLS of Saccharomyces cerevisiae Nab2, J. Struct. Funct. Genom. 14 (2013) 31-35.

[52] D. Dormann, T. Madl, C.F. Valori, E. Bentmann, S. Tahirovic, C. Abou-Ajram, et al., Arginine methylation next to the PY-NLS modulates transportin binding and nuclear import of FUS, EMBO J. 31 (2012) 4258-4275.

[53] Y.M. Chook, A. Jung, M.K. Rosen, G. Blobel, Uncoupling Kapbeta2 substrate dissociation and ran binding, Biochemistry 41 (2002) 6955-6966.

[54] Y.M. Chook, G. Blobel, Structure of the nuclear transport complex karyopherin-beta2-RanGppNHp, Nature 399 (1999) 230-237.

[55] N. Kataoka, J.L. Bachorik, G. Dreyfuss, Transportin-SR, a nuclear import receptor for SR proteins, J. Cell Biol. 145 (1999) 1145-1152.

[56] M.C. Lai, R.I. Lin, S.Y. Huang, C.W. Tsai, W.Y. Tarn, A human importin-beta family protein, transportin-SR2, interacts with the phosphorylated RS domain of SR proteins, J. Biol. Chem. 275 (2000) 7950-7957.
[57] M.C. Lai, R.I. Lin, W.Y. Tarn, Transportin-SR2 mediates nuclear import of phosphorylated SR proteins, Proc. Natl. Acad. Sci. U. S. A. 98 (2001) 10154-10159.

[58] G.N. Maertens, N.J. Cook, W. Wang, S. Hare, S.S. Gupta, I. Oztop, et al., Structural basis for nuclear import of splicing factors by human Transportin 3, Proc. Natl. Acad. Sci. U. S. A. 111 (2014) 2728-2733.

[59] J.M. Mingot, S. Kostka, R. Kraft, E. Hartmann, D. Gorlich, Importin 13: A novel mediator of nuclear import and export, EMBO J. 20 (2001) 3685-3694.

[60] F. Bono, A.G. Cook, M. Grunwald, J. Ebert, E. Conti, Nuclear import mechanism of the EJC component MagoY14 revealed by structural studies of importin 13, Mol. Cell 37 (2010) 211-222.

[61] M. Grunwald, F. Bono, Structure of Importin13-Ubc9 complex: Nuclear import and release of a key regulator of sumoylation, EMBO J. 30 (2011) 427-438.

[62] M. Grunwald, D. Lazzaretti, F. Bono, Structural basis for the nuclear export activity of Importin13, EMBO J. 32 (2013) 899-913.

[63] D. Gorlich, S. Kostka, R. Kraft, C. Dingwall, R.A. Laskey, E. Hartmann, et al., Two different subunits of importin cooperate to recognize nuclear localization signals and bind them to the nuclear envelope, Curr. Biol. 5 (1995) 383-392.

[64] A. Lange, R.E. Mills, C.J. Lange, M. Stewart, S.E. Devine, A.H. Corbett, Classical nuclear localization signals: Definition, function, and interaction with importin alpha, J. Biol. Chem. 282 (2007) 5101-5105.

[65] M. Marfori, A. Mynott, J.J. Ellis, A.M. Mehdi, N.F. Saunders, P.M. Curmi, et al., Molecular basis for specificity of nuclear import and prediction of nuclear localization, Biochim. Biophys. Acta 2011 (1813) 1562-1577.

[66] C.W. Chang, R.M. Counago, S.J. Williams, M. Boden, B. Kobe, The distribution of different classes of nuclear localization signals (NLSs) in diverse organisms and the utilization of the minor NLS-binding site inplantnuclear import factor importin-alpha, Plant Signal. Behav. (2013), http://dx.doi.org/10.4161/psb.25976.

[67] D. Kalderon, W.D. Richardson, A.F. Markham, A.E. Smith, Sequence requirements for nuclear location of simian virus 40 large-T antigen, Nature 311 (1984) 33-38.

[68] D. Kalderon, B.L. Roberts, W.D. Richardson, A.E. Smith, A short amino acid sequence able to specify nuclear location, Cell 39 (1984) 499-509.

[69] C. Dingwall, J. Robbins, S.M. Dilworth, B. Roberts, W.D. Richardson, The nucleoplasmin nuclear location sequence is larger and more complex than that of SV-40 large T antigen, J. Cell Biol. 107 (1988) 841-849.

[70] J. Robbins, S.M. Dilworth, R.A. Laskey, C. Dingwall, Two interdependent basic domains in nucleoplasmin nuclear targeting sequence: Identification of a class of bipartite nuclear targeting sequence, Cell 64 (1991) 615-623.

[71] S.A. Adam, R.S. Marr, L. Gerace, Nuclear protein import in permeabilized mammalian cells requires soluble cytoplasmic factors, J. Cell Biol. 111 (1990) 807-816.

[72] E.J. Adam, S.A. Adam, Identification of cytosolic factors required for nuclear location sequence-mediated binding to the nuclear envelope, J. Cell Biol. 125 (1994) 547-555.

[73] D. Gorlich, S. Prehn, R.A. Laskey, E. Hartmann, Isolation of a protein that is essential for the first step of nuclear protein import, Cell 79 (1994) 767-778.

[74] N.C. Chi, E.J. Adam, S.A. Adam, Sequence and characterization of cytoplasmic nuclear protein import factor p97, J. Cell Biol. 130 (1995) 265-274. 
[75] N. Imamoto, T. Shimamoto, S. Kose, T. Takao, T. Tachibana, M. Matsubae, et al., The nuclear pore targeting complex binds to nuclear pores after association with a karyophile, FEBS Lett. 368 (1995) 415-419.

[76] A. Radu, G. Blobel, M.S. Moore, Identification of a protein complex that is required for nuclear protein import and mediates docking of import substrate to distinct nucleoporins, Proc. Natl. Acad. Sci. U. S. A. 92 (1995) 1769-1773.

[77] K. Weis, U. Ryder, A.I. Lamond, The conserved aminoterminal domain of hSRP1 alpha is essential for nuclear protein import, EMBO J. 15 (1996) 1818-1825.

[78] B. Kobe, J. Heierhorst, S.C. Feil, M.W. Parker, G.M. Benian, K.R. Weiss, et al., Giant protein kinases: Domain interactions and structural basis of autoregulation, EMBO J. 15 (1996) 6810-6821.

[79] E. Conti, M. Uy, L. Leighton, G. Blobel, J. Kuriyan, Crystallographic analysis of the recognition of a nuclear localization signal by the nuclear import factor karyopherin alpha, Cell 94 (1998) 193-204.

[80] B. Riggleman, E. Wieschaus, P. Schedl, Molecular analysis of the armadillo locus: Uniformly distributed transcripts and a protein with novel internal repeats are associated with a Drosophila segment polarity gene, Genes Dev. 3 (1989) 96-113.

[81] S.M. Dias, K.F. Wilson, K.S. Rojas, A.L. Ambrosio, R.A. Cerione, The molecular basis for the regulation of the capbinding complex by the importins, Nat. Struct. Mol. Biol. 16 (2009) 930-937.

[82] S. Jeong, K. Kim, J.H. Lee, J.S. Cha, P. Khadka, H.S. Cho, et al., Akt-mediated phosphorylation increases the binding affinity of hTERT for importin a to promote nuclear translocation, J. Cell Sci. 128 (2015) 2287-2301.

[83] F. Tarendeau, J. Boudet, D. Guilligay, P.J. Mas, C.M. Bougault, S. Boulo, et al., Structure and nuclear import function of the $\mathrm{C}$-terminal domain of influenza virus polymerase PB2 subunit, Nat. Struct. Mol. Biol. 14 (2007) 229-233.

[84] R.A. Pumroy, S. Ke, D.J. Hart, U. Zachariae, G. Cingolani, Molecular determinants for nuclear import of influenza $A$ PB2 by importin alpha isoforms 3 and 7, Structure 23 (2015) 374-384.

[85] S. Trowitzsch, C. Viola, E. Scheer, S. Conic, V. Chavant, M. Fournier, et al., Cytoplasmic TAF2-TAF8-TAF10 complex provides evidence for nuclear holo-TFIID assembly from preformed submodules, Nat. Commun. 6 (2015) 6011.

[86] Y. Matsuura, A. Lange, M.T. Harreman, A.H. Corbett, M. Stewart, Structural basis for Nup2p function in cargo release and karyopherin recycling in nuclear import, EMBO J. 22 (2003) 5358-5369.

[87] M.R. Fontes, T. Teh, B. Kobe, Structural basis of recognition of monopartite and bipartite nuclear localization sequences by mammalian importin-alpha, J. Mol. Biol. 297 (2000) 1183-1194.

[88] M.R. Fontes, T. Teh, D. Jans, R.I. Brinkworth, B. Kobe, Structural basis for the specificity of bipartite nuclear localization sequence binding by importin-alpha, J. Biol. Chem. 278 (2003) 27981-27987.

[89] M.R. Fontes, T. Teh, G. Toth, A. John, I. Pavo, D.A. Jans, et al., Role of flanking sequences and phosphorylation in the recognition of the simian-virus- 40 large T-antigen nuclear localization sequences by importin-alpha, Biochem. J. 375 (2003) 339-349.

[90] M. Marfori, T.G. Lonhienne, J.K. Forwood, B. Kobe, Structural basis of high-affinity nuclear localization signal interactions with importin-alpha, Traffic 13 (2012) 532-548.
[91] A. Giesecke, M. Stewart, Novel binding of the mitotic regulator TPX2 (target protein for Xenopus kinesin-like protein 2) to importin-alpha, J. Biol. Chem. 285 (2010) 17628-17635.

[92] N. Roman, M. Christie, C.M. Swarbrick, B. Kobe, J.K. Forwood, Structural characterisation of the nuclear import receptor importin alpha in complex with the bipartite NLS of Prp20, PLoS One 8 (2013) e82038.

[93] G. Rona, M. Marfori, M. Borsos, I. Scheer, E. Takacs, J. Toth, et al., Phosphorylation adjacent to the nuclear localization signal of human dUTPase abolishes nuclear import: Structural and mechanistic insights, Acta Crystallogr. D Biol. Crystallogr. 69 (2013) 2495-2505.

[94] M.L. Cutress, H.C. Whitaker, I.G. Mills, M. Stewart, D.E. Neal, Structural basis for the nuclear import of the human androgen receptor, J. Cell Sci. 121 (2008) 957-968.

[95] M.H. Chen, I. Ben-Efraim, G. Mitrousis, N. Walker-Kopp, P.J. Sims, G. Cingolani, Phospholipid scramblase 1 contains a nonclassical nuclear localization signal with unique binding site in importin alpha, J. Biol. Chem. 280 (2005) 10599-10606.

[96] A.A. Takeda, A.C. de Barros, C.W. Chang, B. Kobe, M.R. Fontes, Structural basis of importin-alpha-mediated nuclear transport for Ku70 and Ku80, J. Mol. Biol. 412 (2011) 226-234.

[97] S.N. Yang, A.A. Takeda, M.R. Fontes, J.M. Harris, D.A. Jans, B. Kobe, Probing the specificity of binding to the major nuclear localization sequence-binding site of importin\{alpha\} using oriented peptide library screening, J. Biol. Chem. 285 (2010) 19935-19946.

[98] A.V. Mynott, S.J. Harrop, L.J. Brown, S.N. Breit, B. Kobe, P.M. Curmi, Crystal structure of importin-alpha bound to a peptide bearing the nuclear localisation signal from chloride intracellular channel protein 4, FEBS J. 278 (2011) 1662-1675.

[99] K. Lott, A. Bhardwaj, P.J. Sims, G. Cingolani, A minimal nuclear localization signal (NLS) in human phospholipid scramblase 4 that binds only the minor NLS-binding site of importin alpha1, J. Biol. Chem. 286 (2011) 28160-28169.

[100] C.W. Chang, R.L. Counago, S.J. Williams, M. Boden, B. Kobe, Crystal structure of rice importin-alpha and structural basis of its interaction with plant-specific nuclear localization signals, Plant Cell 24 (2012) 5074-5088.

[101] C.W. Chang, R.M. Counago, S.J. Williams, M. Boden, B. Kobe, Distinctive conformation of minor site-specific nuclear localization signals bound to importin-alpha, Traffic 14 (2013) 1144-1154.

[102] Y. Matsuura, M. Stewart, Nup50/Npap60 function in nuclear protein import complex disassembly and importin recycling, EMBO J. 24 (2005) 3681-3689.

[103] C.W. Chang, S.J. Williams, R.M. Counago, B. Kobe, Structural basis of interaction of bipartite nuclear localization signal from Agrobacterium VirD2 with rice importinalpha, Mol. Plant 7 (2014) 1061-1064.

[104] L. Wirthmueller, C. Roth, G. Fabro, M.C. Caillaud, G. Rallapalli, S. Asai, et al., Probing formation of cargo/importin-alpha transport complexes in plant cells using a pathogen effector, Plant J. 81 (2015) 40-52.

[105] N.E. Bernardes, A.A. Takeda, T.R. Dreyer, F.Z. Freitas, M.C. Bertolini, M.R. Fontes, Structure of importin-alpha from a filamentous fungus in complex with a classical nuclear localization signal, PLoS One 10 (2015) e0128687.

[106] S.W. Leung, M.T. Harreman, M.R. Hodel, A.E. Hodel, A.H. Corbett, Dissection of the karyopherin alpha nuclear localization signal (NLS)-binding groove: Functional 
requirements for NLS binding, J. Biol. Chem. 278 (2003) 41947-41953.

[107] M.R. Hodel, A.H. Corbett, A.E. Hodel, Dissection of a nuclear localization signal, J. Biol. Chem. 276 (2001) 1317-1325.

[108] W.H. Colledge, W.D. Richardson, M.D. Edge, A.E. Smith, Extensive mutagenesis of the nuclear location signal of simian virus 40 large-T antigen, Mol. Cell. Biol. 6 (1986) 4136-4139.

[109] J.P.S. Makkerh, C. Dingwall, R.A. Laskey, Comparative mutagenesis of nuclear localization signals reveals the importance of neutral and acidic amino acids, Curr. Biol. 6 (1996) 1025-1027.

[110] S. Kosugi, M. Hasebe, T. Entani, S. Takayama, M. Tomita, H. Yanagawa, Design of peptide inhibitors for the importin alpha/beta nuclear import pathway by activity-based profiling, Chem. Biol. 15 (2008) 940-949.

[111] S. Kosugi, M. Hasebe, N. Matsumura, H. Takashima, E. Miyamoto-Sato, M. Tomita, et al., Six classes of nuclear localization signals specific to different binding grooves of importin alpha, J. Biol. Chem. 284 (2009) 478-485.

[112] T. Wolff, G. Unterstab, G. Heins, J.A. Richt, M. Kann, Characterization of an unusual importin a binding motif in the Borna disease virus p10 protein that directs nuclear import, J. Biol. Chem. 277 (2002) 12151-12157.

[113] W. Xu, M.R. Edwards, D.M. Borek, A.R. Feagins, A. Mittal, J.B. Alinger, et al., Ebola virus VP24 targets a unique NLS binding site on karyopherin alpha 5 to selectively compete with nuclear import of phosphorylated STAT1, Cell Host Microbe 16 (2014) 187-200.

[114] P. Fanara, M.R. Hodel, A.H. Corbett, A.E. Hodel, Quantitative analysis of nuclear localization signal (NLS)-importin alpha interaction through fluorescence depolarization. Evidence for auto-inhibitory regulation of NLS binding, J. Biol. Chem. 275 (2000) 21218-21223.

[115] M.T. Harreman, P.E. Cohen, M.R. Hodel, G.J. Truscott, A.H. Corbett, A.E. Hodel, Characterization of the autoinhibitory sequence within the N-terminal domain of importin alpha, J. Biol. Chem. 278 (2003) 21361-21369.

[116] Y. Matsuura, M. Stewart, Structural basis for the assembly of a nuclear export complex, Nature 432 (2004) 872-877.

[117] R.K. Lokareddy, R.A. Hapsari, M. van Rheenen, R.A. Pumroy, A. Bhardwaj, A. Steen, et al., Distinctive properties of the nuclear localization signals of inner nuclear membrane proteins Heh1 and Heh2, Structure 23 (2015) 1305-1316.

[118] M. Kohler, C. Speck, M. Christiansen, F.R. Bischoff, S. Prehn, H. Haller, et al., Evidence for distinct substrate specificities of importin alpha members in nuclear protein import, Mol. Cell. Biol. 19 (1999) 7782-7791.

[119] D.S. Goldfarb, A.H. Corbett, D.A. Mason, M.T. Harreman, S.A. Adam, Importin alpha: A multipurpose nuclear-transport receptor, Trends Cell Biol. 14 (2004) 505-514.

[120] D.A. Mason, D.E. Stage, D.S. Goldfarb, Evolution of the metazoan-specific importin alpha gene family, J. Mol. Evol. 68 (2009) 351-365.

[121] D.A. Mason, R.J. Fleming, D.S. Goldfarb, Drosophila melanogaster importin alpha1 and alpha3 can replace importin alpha2 during spermatogenesis but not oogenesis, Genetics 161 (2002) 157-170.

[122] F. Rother, T. Shmidt, E. Popova, A. Krivokharchenko, S. Hugel, L. Vilianovich, et al., Importin alpha7 is essential for zygotic genome activation and early mouse development, PLoS One 6 (2011) e18310.
[123] B. Talcott, M.S. Moore, The nuclear import of RCC1 requires a specific nuclear localization sequence receptor, karyopherin alpha3/Qip, J. Biol. Chem. 275 (2000) 10099-10104.

[124] T. Sekimoto, N. Imamoto, K. Nakajima, T. Hirano, Y. Yoneda, Extracellular signal-dependent nuclear import of Stat1 is mediated by nuclear pore-targeting complex formation with NPI-1, but not Rch1, EMBO J. 16 (1997) 7067-7077.

[125] J. Ma, X. Cao, Regulation of Stat3 nuclear import by importin alpha5 and importin alpha7 via two different functional sequence elements, Cell. Signal. 18 (2006) 1117-1126.

[126] R.A. Pumroy, J.D. Nardozzi, D.J. Hart, M.J. Root, G. Cingolani, Nucleoporin Nup50 stabilizes closed conformation of armadillo repeat 10 in importin alpha5, J. Biol. Chem. 287 (2012) 2022-2031.

[127] D. Gilchrist, B. Mykytka, M. Rexach, Accelerating the rate of disassembly of karyopherin-cargo complexes, J. Biol. Chem. 277 (2002) 18161-18172.

[128] U. Kutay, F.R. Bischoff, S. Kostka, R. Kraft, D. Gorlich, Export of importin alpha from the nucleus is mediated by a specific nuclear transport factor, Cell 90 (1997) 1061-1071.

[129] D. Gorlich, M. Dabrowski, F.R. Bischoff, U. Kutay, P. Bork, E. Hartmann, et al., A novel class of RanGTP binding proteins, J. Cell Biol. 138 (1997) 65-80.

[130] D.M. Koepp, D.H. Wong, A.H. Corbett, P.A. Silver, Dynamic localization of the nuclear import receptor and its interactions with transport factors, J. Cell Biol. 133 (1996) 1163-1176.

[131] J. Huber, J. Cronshagen, M. Kadokura, C. Marshallsay, T. Wada, M. Sekine, et al., Snurportin1, an $\mathrm{m}_{3} \mathrm{G}$-cap-specific nuclear import receptor with a novel domain structure, EMBO J. 17 (1998) 4114-4126.

[132] A. Strasser, A. Dickmanns, R. Luhrmann, R. Ficner, Structural basis for m3G-cap-mediated nuclear import of spliceosomal UsnRNPs by snurportin1, EMBO J. 24 (2005) 2235-2243.

[133] D. Kressler, G. Bange, Y. Ogawa, G. Stjepanovic, B. Bradatsch, D. Pratte, et al., Synchronizing nuclear import of ribosomal proteins with ribosome assembly, Science 338 (2012) 666-671.

[134] F.R. Calvino, S. Kharde, A. Ori, A. Hendricks, K. Wild, D. Kressler, et al., Symportin 1 chaperones 5S RNP assembly during ribosome biogenesis by occupying an essential rRNA-binding site, Nat. Commun. 6 (2015) 6510.

[135] K. Van Impe, T. Hubert, V. De Corte, B. Vanloo, C. Boucherie, J. Vandekerckhove, et al., A new role for nuclear transport factor 2 and Ran: Nuclear import of CapG, Traffic 9 (2008) 695-707.

[136] M. Lu, J. Zak, S. Chen, L. Sanchez-Pulido, D.T. Severson, J. Endicott, et al., A code for RanGDP binding in ankyrin repeats defines a nuclear import pathway, Cell 157 (2014) 1130-1145.

[137] T.D. Sweitzer, J.A. Hanover, Calmodulin activates nuclear protein import: A link between signal transduction and nuclear transport, Proc. Natl. Acad. Sci. U. S. A. 93 (1996) 14574-14579.

[138] J.A. Hanover, D.C. Love, N. DeAngelis, M.E. O'Kane, R. Lima-Miranda, T. Schulz, et al., The high mobility group box transcription factor Nhp6Ap enters the nucleus by a calmodulin-dependent, Ran-independent pathway, J. Biol. Chem. 282 (2007) 33743-33751.

[139] A. Argentaro, H. Sim, S. Kelly, S. Preiss, A. Clayton, D.A. Jans, et al., A SOX9 defect of calmodulin-dependent nuclear import in campomelic dysplasia/autosomal sex reversal, J. Biol. Chem. 278 (2003) 33839-33847. 
[140] M. Sharma, M. Johnson, M. Brocardo, C. Jamieson, B.R. Henderson, Wnt signaling proteins associate with the nuclear pore complex: Implications for cancer, Adv. Exp. Med. Biol. 773 (2014) 353-372.

[141] C. Klose, A. Viczian, S. Kircher, E. Schafer, F. Nagy, Molecular mechanisms for mediating light-dependent nucleo/cytoplasmic partitioning of phytochrome photoreceptors, New Phytol. 206 (2015) 965-971.

[142] D.A. Jans, L.J. Briggs, S.E. Gustin, P. Jans, S. Ford, I.G. Young, The cytokine interleukin-5 (IL-5) effects cotransport of its receptor subunits to the nucleus in vitro, FEBS Lett. 410 (1997) 368-372.

[143] M.E. Liku, V.Q. Nguyen, A.W. Rosales, K. Irie, J.J. Li, CDK phosphorylation of a novel NLS-NES module distributed between two subunits of the Mcm2-7 complex prevents chromosomal rereplication, Mol. Biol. Cell 16 (2005) 5026-5039.

[144] S. Steidl, A. Tuncher, H. Goda, C. Guder, N. Papadopoulou, T. Kobayashi, et al., A single subunit of a heterotrimeric CCAAT-binding complex carries a nuclear localization signal: Piggy back transport of the pre-assembled complex to the nucleus, J. Mol. Biol. 342 (2004) 515-524.

[145] N. Zhang, P. Wei, A. Gong, W.T. Chiu, H.T. Lee, H. Colman, et al., FoxM1 promotes beta-catenin nuclear localization and controls Wht target-gene expression and glioma tumorigenesis, Cancer Cell 20 (2011) 427-442.

[146] M. Baake, M. Bauerle, D. Doenecke, W. Albig, Core histones and linker histones are imported into the nucleus by different pathways, Eur. J. Cell Biol. 80 (2001) 669-677.

[147] M. Baake, D. Doenecke, W. Albig, Characterisation of nuclear localisation signals of the four human core histones, J. Cell. Biochem. 81 (2001) 333-346.

[148] N. Mosammaparast, Y. Guo, J. Shabanowitz, D.F. Hunt, L.F. Pemberton, Pathways mediating the nuclear import of histones H3 and H4 in yeast, J. Biol. Chem. 277 (2002) 862-868.

[149] N. Mosammaparast, L.F. Pemberton, Karyopherins: From nuclear-transport mediators to nuclear-function regulators, Trends Cell Biol. 14 (2004) 547-556.

[150] K.M. Wagstaff, D.A. Jans, Importins and beyond: Nonconventional nuclear transport mechanisms, Traffic 10 (2009) 1188-1198.

[151] S. Kose, M. Furuta, N. Imamoto, Hikeshi, a nuclear import carrier for Hsp70s, protects cells from heat shock-induced nuclear damage, Cell 149 (2012) 578-589.

[152] J. Song, S. Kose, A. Watanabe, S.Y. Son, S. Choi, H. Hong, et al., Structural and functional analysis of Hikeshi, a new nuclear transport receptor of Hsp70s, Acta Crystallogr. D Biol. Crystallogr. 71 (2015) 473-483.

[153] K. Ribbeck, G. Lipowsky, H.M. Kent, M. Stewart, D. Gorlich, NTF2 mediates nuclear import of Ran, EMBO J. 17 (1998) 6587-6598.

[154] D.H. Wong, A.H. Corbett, H.M. Kent, M. Stewart, P.A. Silver, Interaction between the small GTPase Ran/Gsp1p and Ntf2p is required for nuclear transport, Mol. Cell. Biol. 17 (1997) 3755-3767.

[155] M. Stewart, Insights into the molecular mechanism of nuclear trafficking using nuclear transport factor 2 (NTF2), Cell Struct. Funct. 25 (2000) 217-225.

[156] T.L. Bullock, W.D. Clarkson, H.M. Kent, M. Stewart, The 1.6 angstroms resolution crystal structure of nuclear transport factor 2 (NTF2), J. Mol. Biol. 260 (1996) 422-431.

[157] C. Chaillan-Huntington, C.V. Braslavsky, J. Kuhlmann, M. Stewart, Dissecting the interactions between NTF2, RanGDP, and the nucleoporin XFXFG repeats, J. Biol. Chem. 275 (2000) 5874-5879.

[158] M. Stewart, H.M. Kent, A.J. McCoy, Structural basis for molecular recognition between nuclear transport factor 2 (NTF2) and the GDP-bound form of the Ras-family GTPase Ran, J. Mol. Biol. 277 (1998) 635-646.

[159] R. Bayliss, K. Ribbeck, D. Akin, H.M. Kent, C.M. Feldherr, D. Gorlich, et al., Interaction between NTF2 and xFxFGcontaining nucleoporins is required to mediate nuclear import of RanGDP, J. Mol. Biol. 293 (1999) 579-593.

[160] I.K. Poon, D.A. Jans, Regulation of nuclear transport: Central role in development and transformation? Traffic 6 (2005) 173-186.

[161] C.W. Pouton, K.M. Wagstaff, D.M. Roth, G.W. Moseley, D.A. Jans, Targeted delivery to the nucleus, Adv. Drug Deliv. Rev. 59 (2007) 698-717.

[162] L.M. McLane, A.H. Corbett, Nuclear localization signals and human disease, IUBMB Life 61 (2009) 697-706.

[163] A.E. Hodel, M.T. Harreman, K.F. Pulliam, M.E. Harben, J.S. Holmes, M.R. Hodel, et al., Nuclear localization signal receptor affinity correlates with in vivo localization in Saccharomyces cerevisiae, J. Biol. Chem. 281 (2006) 23545-23556.

[164] B.L. Timney, J. Tetenbaum-Novatt, D.S. Agate, R. Williams, W. Zhang, B.T. Chait, et al., Simple kinetic relationships and nonspecific competition govern nuclear import rates in vivo, J. Cell Biol. 175 (2006) 579-593.

[165] K.K. Biggar, S.S. Li, Non-histone protein methylation as a regulator of cellular signalling and function, Nat. Rev. Mol. Cell Biol. 16 (2015) 5-17.

[166] K. Sadoul, J. Wang, B. Diagouraga, S. Khochbin, The tale of protein lysine acetylation in the cytoplasm, J. Biomed. Biotechnol. 2011 (2011) 970382.

[167] E. Verdin, M. Ott, 50 years of protein acetylation: From gene regulation to epigenetics, metabolism and beyond, Nat. Rev. Mol. Cell Biol. 16 (2015) 258-264.

[168] H. Wei, R. Mundade, K.C. Lange, T. Lu, Protein arginine methylation of non-histone proteins and its role in diseases, Cell Cycle 13 (2014) 32-41.

[169] X. Zhang, H. Wen, X. Shi, Lysine methylation: Beyond histones, Acta Biochim. Biophys. Sin. (Shanghai) 44 (2012) 14-27.

[170] D.A. Jans, The regulation of protein transport to the nucleus by phosphorylation, Biochem. J. 311 (1995) 705-716.

[171] D.A. Jans, S. Hubner, Regulation of protein transport to the nucleus: Central role of phosphorylation, Physiol. Rev. 76 (1996) 651-685.

[172] J.D. Nardozzi, K. Lott, G. Cingolani, Phosphorylation meets nuclear import: A review, Cell Commun Signal. 8 (2010) 32.

[173] A. Kaffman, N.M. Rank, E.K. O'Shea, Phosphorylation regulates association of the transcription factor $\mathrm{Pho} 4$ with its import receptor Pse1/Kap121, Genes Dev. 12 (1998) 2673-2683.

[174] A. Komeili, E.K. O'Shea, Roles of phosphorylation sites in regulating activity of the transcription factor Pho4, Science 284 (1999) 977-980.

[175] G. Rona, M. Borsos, J.J. Ellis, A.M. Mehdi, M. Christie, Z. Kornyei, et al., Dynamics of re-constitution of the human nuclear proteome after cell division is regulated by NLSadjacent phosphorylation, Cell Cycle 13 (2014) 3551-3564.

[176] S. Kosugi, M. Hasebe, M. Tomita, H. Yanagawa, Systematic identification of cell cycle-dependent yeast nucleocytoplasmic shuttling proteins by prediction of composite motifs, Proc. Natl. Acad. Sci. U. S. A. 106 (2009) 10171-10176. 
[177] M.T. Harreman, T.M. Kline, H.G. Milford, M.B. Harben, A.E. Hodel, A.H. Corbett, Regulation of nuclear import by phosphorylation adjacent to nuclear localization signals, J. Biol. Chem. 279 (2004) 20613-20621.

[178] S. Hübner, C.Y. Xiao, D.A. Jans, The protein kinase CK2 site (Ser111/112) enhances recognition of the simian virus 40 large $\mathrm{T}$-antigen nuclear localization sequence by importin, J. Biol. Chem. 272 (1997) 17191-17195.

[179] R. Kitamura, T. Sekimoto, S. Ito, S. Harada, H. Yamagata, $\mathrm{H}$. Masai, et al., Nuclear import of Epstein-Barr virus nuclear antigen 1 mediated by NPI-1 (Importin alpha5) is up- and down-regulated by phosphorylation of the nuclear localization signal for which Lys379 and Arg380 are essential, J. Virol. 80 (2006) 1979-1991.

[180] J. Nardozzi, N. Wenta, N. Yasuhara, U. Vinkemeier, G. Cingolani, Molecular basis for the recognition of phosphorylated STAT1 by importin alpha5, J. Mol. Biol. 402 (2010) 83-100.

[181] G. Humbert-Lan, T. Pieler, Regulation of DNA binding activity and nuclear transport of B-Myb in Xenopus oocytes, J. Biol. Chem. 274 (1999) 10293-10300.

[182] R. Fagerlund, K. Mélen, L. Kinnunen, I. Julkunen, Arginine/ lysine-rich nuclear localization signals mediate interactions between dimeric STATs and importin alpha 5, J. Biol. Chem. 277 (2002) 30072-30078.

[183] K.M. McBride, G. Banninger, C. McDonald, N.C. Reich, Regulated nuclear import of the STAT1 transcription factor by direct binding of importin-alpha, EMBO J. 21 (2002) 1754-1763.

[184] N. Wenta, H. Strauss, S. Meyer, U. Vinkemeier, Tyrosine phosphorylation regulates the partitioning of STAT1 between different dimer conformations, Proc. Natl. Acad. Sci. U. S. A. 105 (2008) 9238-9243.

[185] T. Meyer, A. Begitt, I. Lodige, M. van Rossum, U. Vinkemeier, Constitutive and IFN-gamma-induced nuclear import of STAT1 proceed through independent pathways, EMBO J. 21 (2002) 344-354.

[186] T. Huxford, D.-B. Huang, S. Malek, G. Ghosh, The crystal structure of the IkappaBalpha/NF-kappaB complex reveals mechanisms of NF-kappaB inactivation, Cell 95 (1998) 759-770.

[187] M.D. Jacobs, S.C. Harrison, Structure of an IkappaBalpha/ NF-kappaB complex, Cell 95 (1998) 749-758.

[188] R. Fagerlund, L. Kinnunen, M. Kohler, I. Julkunen, K. Melen, NF-\{kappa\}B is transported into the nucleus by importin \{alpha\}3 and importin \{alpha\}4, J. Biol. Chem. 280 (2005) 15942-15951.

[189] A.A. Beg, S.M. Ruben, R.I. Scheinman, S. Haskill, C.A. Rosen, A.S. Baldwin Jr., I kappa B interacts with the nuclear localization sequences of the subunits of NF-kappa B: A mechanism for cytoplasmic retention, Genes Dev. 6 (1992) 1899-1913.

[190] T. Henkel, T. Machleidt, I. Alkalay, M. Kronke, Y. BenNeriah, P.A. Baeuerle, Rapid proteolysis of I kappa B-alpha is necessary for activation of transcription factor NF-kappa B, Nature 365 (1993) 182-185.

[191] A. Oeckinghaus, M.S. Hayden, S. Ghosh, Crosstalk in NF-kappaB signaling pathways, Nat. Immunol. 12 (2011) 695-708.

[192] M. Asada, K. Ohmi, D. Delia, S. Enosawa, S. Suzuki, A. Yuo, et al., Brap2 functions as a cytoplasmic retention protein for p21 during monocyte differentiation, Mol. Cell. Biol. 24 (2004) 8236-8243.

[193] A.J. Fulcher, D.M. Roth, S. Fatima, G. Alvisi, D.A. Jans, The BRCA-1 binding protein BRAP2 is a novel, negative regulator of nuclear import of viral proteins, dependent on phosphorylation flanking the nuclear localization signal, FASEB J. 24 (2010) 1454-1466.

[194] J.K. Forwood, V. Harley, D.A. Jans, The C-terminal nuclear localization signal of the sex-determining region $\mathrm{Y}$ (SRY) high mobility group domain mediates nuclear import through importin beta 1, J. Biol. Chem. 276 (2001) 46575-46582.

[195] L. Thevenet, C. Mejean, B. Moniot, N. Bonneaud, N. Galeotti, G. Aldrian-Herrada, et al., Regulation of human SRY subcellular distribution by its acetylation/deacetylation, EMBO J. 23 (2004) 3336-3345.

[196] T.G. Nishino, M. Miyazaki, H. Hoshino, Y. Miwa, S. Horinouchi, M. Yoshida, 14-3-3 regulates the nuclear import of class Ila histone deacetylases, Biochem. Biophys. Res. Commun. 377 (2008) 852-856.

[197] B.C. Harrison, K. Huynh, G.L. Lundgaard, S.M. Helmke, M.B. Perryman, T.A. McKinsey, Protein kinase C-related kinase targets nuclear localization signals in a subset of class Ila histone deacetylases, FEBS Lett. 584 (2010) 1103-1110.

[198] C. Choudhary, C. Kumar, F. Gnad, M.L. Nielsen, M. Rehman, T.C. Walther, et al., Lysine acetylation targets protein complexes and co-regulates major cellular functions, Science 325 (2009) 834-840.

[199] A. Guo, H. Gu, J. Zhou, D. Mulhern, Y. Wang, K.A. Lee, et al., Immunoaffinity enrichment and mass spectrometry analysis of protein methylation, Mol. Cell. Proteomics 13 (2014) 372-387.

[200] K.E. Moore, S.M. Carlson, N.D. Camp, P. Cheung, R.G. James, K.F. Chua, et al., A general molecular affinity strategy for global detection and proteomic analysis of lysine methylation, Mol. Cell 50 (2013) 444-456.

[201] A.J. Bannister, E.A. Miska, D. Gorlich, T. Kouzarides, Acetylation of importin-alpha nuclear import factors by CBP/ p300, Curr. Biol. 10 (2000) 467-470.

[202] C.M. Ryan, J.C. Harries, K.B. Kindle, H.M. Collins, D.M. Heery, Functional interaction of CREB binding protein (CBP) with nuclear transport proteins and modulation by HDAC inhibitors, Cell Cycle 5 (2006) 2146-2152.

[203] X.J. Yang, Lysine acetylation and the bromodomain: A new partnership for signaling, Bioessays 26 (2004) 1076-1087.

[204] M.G. di Bari, L. Ciuffini, M. Mingardi, R. Testi, S. Soddu, D. Barila, c-Abl acetylation by histone acetyltransferases regulates its nuclear-cytoplasmic localization, EMBO Rep. 7 (2006) 727-733.

[205] T. Dietschy, I. Shevelev, J. Pena-Diaz, D. Huhn, S. Kuenzle, R. Mak, et al., p300-mediated acetylation of the RothmundThomson-syndrome gene product RECQL4 regulates its subcellular localization, J. Cell Sci. 122 (2009) 1258-1267.

[206] T. Bonaldi, F. Talamo, P. Scaffidi, D. Ferrera, A. Porto, A. Bachi, et al., Monocytic cells hyperacetylate chromatin protein HMGB1 to redirect it towards secretion, EMBO J. 22 (2003) 5551-5560.

[207] T. Shimazu, S. Horinouchi, M. Yoshida, Multiple histone deacetylases and the CREB-binding protein regulate premRNA 3 '-end processing, J. Biol. Chem. 282 (2007) 4470-4478.

[208] D.L. Madison, P. Yaciuk, R.P. Kwok, J.R. Lundblad, Acetylation of the adenovirus-transforming protein E1A determines nuclear localization by disrupting association with importin-alpha, J. Biol. Chem. 277 (2002) 38755-38763.

[209] N. Blanco-Garcia, E. Asensio-Juan, X. de la Cruz, M.A. Martinez-Balbas, Autoacetylation regulates P/CAF nuclear localization, J. Biol. Chem. 284 (2009) 1343-1352.

[210] K. Wong, J. Zhang, S. Awasthi, A. Sharma, L. Rogers, E.F. Matlock, et al., Nerve growth factor receptor signaling 
induces histone acetyltransferase domain-dependent nuclear translocation of p300/CREB-binding protein-associated factor and hGCN5 acetyltransferases, J. Biol. Chem. 279 (2004) 55667-55674.

[211] E.H. Song, W. Oh, A. Ulu, H.S. Carr, Y. Zuo, J.A. Frost, Acetylation of the RhoA GEF Net1A controls its subcellular localization and activity, J. Cell Sci. 128 (2015) 913-922.

[212] E. Soutoglou, N. Katrakili, I. Talianidis, Acetylation regulates transcription factor activity at multiple levels, Mol. Cell 5 (2000) 745-751.

[213] S.H. Liang, M.F. Clarke, Regulation of p53 localization, Eur. J. Biochem. 268 (2001) 2779-2783.

[214] A. O'Brate, P. Giannakakou, The importance of p53 location: Nuclear or cytoplasmic zip code? Drug Resist. Updat. 6 (2003) 313-322.

[215] Y. Kawaguchi, A. Ito, E. Appella, T.P. Yao, Charge modification at multiple $\mathrm{C}$-terminal lysine residues regulates p53 oligomerization and its nucleus-cytoplasm trafficking, J. Biol. Chem. 281 (2006) 1394-1400.

[216] J.A. Rodriguez, S.W. Span, C.G. Ferreira, F.A. Kruyt, G. Giaccone, CRM1-mediated nuclear export determines the cytoplasmic localization of the antiapoptotic protein Survivin, Exp. Cell Res. 275 (2002) 44-53.

[217] H. Wang, M.P. Holloway, L. Ma, Z.A. Cooper, M. Riolo, A. Samkari, et al., Acetylation directs survivin nuclear localization to repress STAT3 oncogenic activity, J. Biol. Chem. 285 (2010) 36129-36137.

[218] L. Lv, Y.P. Xu, D. Zhao, F.L. Li, W. Wang, N. Sasaki, et al., Mitogenic and oncogenic stimulation of K433 acetylation promotes PKM2 protein kinase activity and nuclear localization, Mol. Cell 52 (2013) 340-352.

[219] M.J. Oudhoff, S.A. Freeman, A.L. Couzens, F. Antignano, E. Kuznetsova, P.H. Min, et al., Control of the hippo pathway by Set7-dependent methylation of Yap, Dev. Cell 26 (2013) 188-194.

[220] H.S. Cho, T. Shimazu, G. Toyokawa, Y. Daigo, Y. Maehara, S. Hayami, et al., Enhanced HSP70 lysine methylation promotes proliferation of cancer cells through activation of Aurora kinase B, Nat. Commun. 3 (2012) 1072.

[221] W.A. Smith, B.T. Schurter, F. Wong-Staal, M. David, Arginine methylation of RNA helicase a determines its subcellular localization, J. Biol. Chem. 279 (2004) 22795-22798.

[222] L.J. Zhao, T. Subramanian, Y. Zhou, G. Chinnadurai, Acetylation by p300 regulates nuclear localization and function of the transcriptional corepressor CtBP2, J. Biol. Chem. 281 (2006) 4183-4189.

[223] X. Lin, B. Sun, M. Liang, Y.Y. Liang, A. Gast, J. Hildebrand, et al., Opposed regulation of corepressor CtBP by SUMOylation and PDZ binding, Mol. Cell 11 (2003) 1389-1396.

[224] G.M. Riefler, B.L. Firestein, Binding of neuronal nitricoxide synthase (nNOS) to carboxyl-terminal-binding protein (CtBP) changes the localization of CtBP from the nucleus to the cytosol: A novel function for targeting by the PDZ domain of nNOS, J. Biol. Chem. 276 (2001) 48262-48268.

[225] C.J. Barnes, R.K. Vadlamudi, S.K. Mishra, R.H. Jacobson, F. Li, R. Kumar, Functional inactivation of a transcriptional corepressor by a signaling kinase, Nat. Struct. Biol. 10 (2003) 622-628.

[226] A. Pickard, P.P. Wong, D.J. McCance, Acetylation of Rb by $\mathrm{PCAF}$ is required for nuclear localization and keratinocyte differentiation, J. Cell Sci. 123 (2010) 3718-3726.
[227] B.B. Chen, R.K. Mallampalli, Masking of a nuclear signal motif by monoubiquitination leads to mislocalization and degradation of the regulatory enzyme cytidylyltransferase, Mol. Cell. Biol. 29 (2009) 3062-3075.

[228] N.D. Marchenko, W. Hanel, D. Li, K. Becker, N. Reich, U.M. Moll, Stress-mediated nuclear stabilization of p53 is regulated by ubiquitination and importin-alpha3 binding, Cell Death Differ. 17 (2010) 255-267.

[229] J. Howitt, J. Lackovic, L.H. Low, A. Naguib, A. Macintyre, C.P. Goh, et al., Ndfip1 regulates nuclear Pten import in vivo to promote neuronal survival following cerebral ischemia, J. Cell Biol. 196 (2012) 29-36.

[230] L.C. Trotman, X. Wang, A. Alimonti, Z. Chen, J. TeruyaFeldstein, $\mathrm{H}$. Yang, et al., Ubiquitination regulates PTEN nuclear import and tumor suppression, Cell 128 (2007) 141-156.

[231] X. Wang, L.C. Trotman, T. Koppie, A. Alimonti, Z. Chen, Z. Gao, et al., NEDD4-1 is a proto-oncogenic ubiquitin ligase for PTEN, Cell 128 (2007) 129-139.

[232] V. Vethantham, N. Rao, J.L. Manley, Sumoylation regulates multiple aspects of mammalian poly(A) polymerase function, Genes Dev. 22 (2008) 499-511.

[233] J.X. Du, A.B. Bialkowska, B.B. McConnell, V.W. Yang, SUMOylation regulates nuclear localization of Kruppel-like factor 5, J. Biol. Chem. 283 (2008) 31991-32002.

[234] K. Truong, T.D. Lee, B. Li, Y. Chen, Sumoylation of SAE2 C terminus regulates SAE nuclear localization, J. Biol. Chem. 287 (2012) 42611-42619.

[235] D.D. Anderson, J.Y. Eom, P.J. Stover, Competition between sumoylation and ubiquitination of serine hydroxymethyltransferase 1 determines its nuclear localization and its accumulation in the nucleus, J. Biol. Chem. 287 (2012) 4790-4799.

[236] A.J. MacFarlane, D.D. Anderson, P. Flodby, C.A. Perry, R.H. Allen, S.P. Stabler, et al., Nuclear localization of de novo thymidylate biosynthesis pathway is required to prevent uracil accumulation in DNA, J. Biol. Chem. 286 (2011) 44015-44022.

[237] C.F. Woeller, D.D. Anderson, D.M. Szebenyi, P.J. Stover, Evidence for small ubiquitin-like modifier-dependent nuclear import of the thymidylate biosynthesis pathway, J. Biol. Chem. 282 (2007) 17623-17631.

[238] K. Dohner, C.H. Nagel, B. Sodeik, Viral stop-and-go along microtubules: Taking a ride with dynein and kinesins, Trends Microbiol. 13 (2005) 320-327.

[239] P.L. Leopold, K.K. Pfister, Viral strategies for intracellular trafficking: Motors and microtubules, Traffic 7 (2006) 516-523.

[240] P. Giannakakou, D.L. Sackett, Y. Ward, K.R. Webster, M.V. Blagosklonny, T. Fojo, p53 is associated with cellular microtubules and is transported to the nucleus by dynein, Nat. Cell Biol. 2 (2000) 709-717.

[241] D.M. Roth, G.W. Moseley, D. Glover, C.W. Pouton, D.A. Jans, A microtubule-facilitated nuclear import pathway for cancer regulatory proteins, Traffic 8 (2007) 673-686.

[242] M.H. Lam, R.J. Thomas, K.L. Loveland, S. Schilders, M. Gu, T.J. Martin, et al., Nuclear transport of parathyroid hormone (PTH)-related protein is dependent on microtubules, Mol. Endocrinol. 16 (2002) 390-401.

[243] D.M. Roth, G.W. Moseley, C.W. Pouton, D.A. Jans, Mechanism of microtubule-facilitated "fast track" nuclear import, J. Biol. Chem. 286 (2011) 14335-14351.

[244] A.R. Lowe, J.H. Tang, J. Yassif, M. Graf, W.Y. Huang, J.T. Groves, et al., Importin-beta modulates the permeability of 
the nuclear pore complex in a Ran-dependent manner, ELife (2015), http://dx.doi.org/10.7554/eLife.04052.

[245] M. Tanaka, M. Nishi, M. Morimoto, T. Sugimoto, M. Kawata, Yellow fluorescent protein-tagged and cyan fluorescent protein-tagged imaging analysis of glucocorticoid receptor and importins in single living cells, Endocrinology 144 (2003) 4070-4079.

[246] P.C. Echeverria, D. Picard, Molecular chaperones, essential partners of steroid hormone receptors for activity and mobility, Biochim. Biophys. Acta 2010 (1803) 641-649.

[247] L. Ni, R. Llewellyn, C.T. Kesler, J.B. Kelley, A. Spencer, C.J. Snow, et al., Androgen induces a switch from cytoplasmic retention to nuclear import of the androgen receptor, Mol. Cell. Biol. 33 (2013) 4766-4778.

[248] J. Falces, I. Arregi, P.V. Konarev, M.A. Urbaneja, D.I. Svergun, S.G. Taneva, et al., Recognition of nucleoplasmin by its nuclear transport receptor importin alpha/beta: Insights into a complete import complex, Biochemistry 49 (2010) 9756-9769.

[249] G. Rona, H.L. Palinkas, M. Borsos, A. Horvath, I. Scheer, A. Benedek, et al., NLS copy-number variation governs efficiency of nuclear import-Case study on dUTPases, FEBS J. 281 (2014) 5463-5478.

[250] F. Melchior, B. Paschal, J. Evans, L. Gerace, Inhibition of nuclear protein import by nonhydrolyzable analogues of
GTP and identification of the small GTPase Ran/TC4 as an essential transport factor, J. Cell Biol. 123 (1993) 1649-1659.

[251] M.S. Moore, G. Blobel, The GTP-binding protein Ran/TC4 is required for protein import into the nucleus, Nature 365 (1993) 661-663.

[252] S. Jakel, W. Albig, U. Kutay, F.R. Bischoff, K. Schwamborn, D. Doenecke, et al., The importin beta/importin 7 heterodimer is a functional nuclear import receptor for histone $\mathrm{H} 1$, EMBO J. 18 (1999) 2411-2423.

[253] K.A. Dean, O. von Ahsen, D. Gorlich, H.M. Fried, Signal recognition particle protein 19 is imported into the nucleus by importin 8 (RanBP8) and transportin, J. Cell Sci. 114 (2001) 3479-3485.

[254] W. Kuhlbrandt, Biochemistry. The resolution revolution, Science 343 (2014) 1443-1444.

[255] I. Schlichting, Serial femtosecond crystallography: The first five years, IUCrJ 2 (2015) 246-255.

[256] Y. Matsuura, Mechanistic insights from structural analyses of Ran GTPase-driven nuclear export of proteins and RNA, J. Mol. Biol. (2016) 2025-2039 (in this issue). 